Research Article

\title{
Exploring the Pharmacological Mechanism of Duhuo Jisheng Decoction in Treating Osteoporosis Based on Network Pharmacology
}

\author{
Zhencheng Xiong $\mathbb{D}^{1,2}$ Can Zheng $\mathbb{D}^{1,}{ }^{3}$ Yanan Chang, ${ }^{4}$ Kuankuan Liu, ${ }^{4}$ Li Shu, ${ }^{4}$ \\ and Chi Zhang $1,3,4,5,6$ \\ ${ }^{1}$ Institute of Medical Technology, Peking University Health Science Center, Beijing, China \\ ${ }^{2}$ Peking University Third Hospital, Beijing, China \\ ${ }^{3}$ Biomedical Engineering Department, Peking University, Beijing, China \\ ${ }^{4}$ Central Laboratory, Peking University International Hospital, Beijing, China \\ ${ }^{5}$ Department of Orthopedics, Peking University International Hospital, Beijing, China \\ ${ }^{6}$ School of Chinese Materia Medica, Beijing University of Chinese Medicine, Beijing, China \\ Correspondence should be addressed to Chi Zhang; chi.zhang@case.edu
}

Received 6 January 2021; Revised 21 March 2021; Accepted 25 March 2021; Published 7 April 2021

Academic Editor: Jun Jiang

Copyright (c) 2021 Zhencheng Xiong et al. This is an open access article distributed under the Creative Commons Attribution License, which permits unrestricted use, distribution, and reproduction in any medium, provided the original work is properly cited.

\begin{abstract}
Objective. The purpose of this work is to study the mechanism of action of Duhuo Jisheng Decoction (DHJSD) in the treatment of osteoporosis based on the methods of bioinformatics and network pharmacology. Methods. In this study, the active compounds of each medicinal ingredient of DHJSD and their corresponding targets were obtained from TCMSP database. Osteoporosis was treated as search query in GeneCards, MalaCards, DisGeNET, Therapeutic Target Database (TTD), Comparative Toxicogenomics Database (CTD), and OMIM databases to obtain disease-related genes. The overlapping targets of DHJSD and osteoporosis were identified, and then GO and KEGG enrichment analysis were performed. Cytoscape was employed to construct DHJSDcompounds-target genes-osteoporosis network and protein-protein interaction (PPI) network. CytoHubba was utilized to select the hub genes. The activities of binding of hub genes and key components were confirmed by molecular docking. Results. 174 active compounds and their 205 related potential targets were identified in DHJSD for the treatment of osteoporosis, including 10 hub genes (AKT1, ALB, IL6, MAPK3, VEGFA, JUN, CASP3, EGFR, MYC, and EGF). Pathway enrichment analysis of target proteins indicated that osteoclast differentiation, AGE-RAGE signaling pathway in diabetic complications, Wnt signaling pathway, MAPK signaling pathway, PI3K-Akt signaling pathway, JAK-STAT signaling pathway, calcium signaling pathway, and TNF signaling pathway were the specifically major pathways regulated by DHJSD against osteoporosis. Further verification based on molecular docking results showed that the small molecule compounds (Quercetin, Kaempferol, Beta-sitosterol, Beta-carotene, and Formononetin) contained in DHJSD generally have excellent binding affinity to the macromolecular target proteins encoded by the top 10 genes. Conclusion. This study reveals the characteristics of multi-component, multi-target, and multi-pathway of DHJSD against osteoporosis and provides novel insights for verifying the mechanism of DHJSD in the treatment of osteoporosis.
\end{abstract}

\section{Introduction}

Osteoporosis is characterized by low bone mass, impaired bone microstructure, increased bone fragility, and susceptibility to fracture and is also a systemic bone disease [1]. Osteoporosis can occur in different genders and ages, but it is more common in postmenopausal women and elderly men [2]. The serious consequences of osteoporosis are fragility fractures that can occur during minor trauma or daily activities, leading to an increase in disability and mortality [3]. Moreover, the treatment and nursing of osteoporosis and osteoporotic fracture need to invest huge 
manpower and material resources, and the cost is high, resulting in heavy family, social, and economic burden [4]. Therefore, early adoption of scientific prevention strategies and standardized treatment is very necessary. Among these, drug therapies such as bisphosphonates and denosumab are commonly used to treat osteoporosis by inhibiting the development, formation, and survival of osteoclasts [1]. Although these drugs are effective, high-dose or long-term use may cause serious adverse effects, such as gastrointestinal tolerance, atypical long bone fracture, jaw osteonecrosis, and acute renal failure [1]. Therefore, continuing to search for potential drugs with significant efficacy and high safety has become a consistent hot spot for the treatment of osteoporosis.

As we all know, traditional Chinese medicine (TCM) plays an important role in health maintenance in China and other Asian countries [5]. For a long time, TCM has been used as a complementary and alternative treatment option for patients with osteoporosis [6]. Systematic reviews and experimental studies have explored the efficacy and safety of TCM prescriptions in the treatment of osteoporosis, including Duhuo Jisheng Decoction (DHJSD), Xianling Gubao capsules, Liuwei Dihuang Decoction, and Erxian Decoction [2, 7-9].

DHJSD is a TCM recorded in Bei Ji Qian Jin Yao Fang of the Tang Dynasty for the treatment of "Bi Zheng," usually consisting of the following 15 herbs, including Du Huo (Radix Angelicae Pubescentis), Sang Ji Sheng (Herba Taxilli), Qin Jiao (Radix Gentianae Macrophyllae), Fang Feng (Radix Saposhnikoviae), Xi Xin (Herba Asari), Fu Ling (Poria Cocos), Chuan Xiong (Rhizoma Chuanxiong), Bai Shao (Radix Paeoniae Alba), Du Zhong (Cortex Eucommiae Ulmoidis), Ren Shen (Panax Ginseng), Gan Cao (Radix Glycyrrhizae), Dang Gui (Radix Angelicae Sinensis), Niu Xi (Radix Achyranthis Bidentatae), Shu Di Huang (Radix Rehmanniae Preparata), and Rou Gui (Cortex Cinnamomi) [10]. Among them, Du Huo has the good effects of relieving the pain of "Bi Zheng," dispelling cold and dehumidification, nourishing blood and Qi; Xi Xin, Fang Feng, and Qin Jiao dispel rheumatism, relax tendons and muscles, and benefit joints; Sang Ji Sheng, Du Zhong, Rou Gui, and Niu Xi nourish liver and kidney and strengthen bones and muscles; Dang Gui, Bai Shao, Shu Di Huang, and Chuan Xiong promote blood circulation; Ren Shen, Gan Cao, and Fu Ling strengthen spleen and supplement Qi [11]. The combination of the above herbs forms DHJSD, the classic bone injury prescription in TCM, it has the effect of nourishing the liver and kidney, benefiting Qi and blood, and stopping the pain of "Bi Zheng", which obviously improves the microcirculation of the body [2]. According to the theory of Chinese medicine, "Bi Zheng" refers to the symptoms of numbness, soreness, and poor flexion of muscles and joints caused by external factors (wind, dampness, cold, heat) on the body surface, tendons, and veins [12]. Osteoporosis belongs to the category of "Gu Bi" and "Gu Lou" in TCM theory [13]. And the cause of osteoporosis is the deficiency of Qi and blood in the liver and kidney and the loss of nutrition in the tendons and bones, so the treatment should be to nourish the liver and kidney and strengthen the tendons and bones as the primary treatment [14]. Therefore, DHJSD has been widely used in China to treat rheumatoid arthritis, intervertebral disc disease, knee osteoarthritis, and osteoporosis, especially postmenopausal osteoporosis [2, 11, 14-17]. However, due to the ingredients contained, the mechanism of action of TCM is often elusive.

The network pharmacology of TCM is a part of bioinformatics, and it is still a priori analysis method for studying the relationship between drugs, compounds, diseases, and targets [18]. Due to the characteristics of multicomponent, multi-target, and multi-pathway of TCM, network pharmacology has been widely used to clarify the mechanism of action of TCM and provide researchers with new directions and strategies. In this work, we tried to use the network pharmacology method to reveal the active compounds of DHJSD, and the key genes and pathways of DHJSD against osteoporosis, which facilitated further research and development (Figure 1).

\section{Materials and Methods}

2.1. Screening of Potential Active Compounds and Related Targets in DHJSD. We used the Traditional Chinese Medicine Systems Pharmacology (TCMSP, Version: 2.3, https:// tcmspw.com/tcmsp.php) database and the analysis platform [19], and input the names of 15 Chinese herbal medicines in DHJSD to obtain the corresponding compounds and related information. According to the absorption, distribution, metabolism, and excretion (ADME) protocols, the active compounds were screened, and the criteria were oral bioavailability $(\mathrm{OB}) \geq 30$ and drug-likeness $(\mathrm{DL}) \geq 0.18[20,21]$. Then, the potential target proteins of the selected active compounds were mined in TCSMP database to construct the potential target gene set of DHJSD. UniProt database (https://www.uniprot.org/) was used to obtain the unique corresponding gene names and UniProt ID of drug or disease-related targets [22]. The species was selected as "Homo sapiens."

\subsection{Mining of Osteoporosis-Related Targets.} Osteoporosis-related targets were obtained through retrieving GeneCards (https://www.genecards.org/) [23], MalaCards (https://www.malacards.org/) [24], DisGeNet database (https://www.disgenet.org/, v7.0) [25], Therapeutic Target Database (TTD) (http://db.idrblab.net/ttd/) [26], Comparative Toxicogenomics Database (CTD) (http:// ctdbase.org/, Last update by June, 2020) [27], and Online Mendelian Inheritance in Man (OMIM) (https://omim.org/, updated November 25, 2020) [28] using the keyword "osteoporosis." GeneCards is a comprehensive, user-friendly database providing information on all annotated and predicted human genes, and we screen out targets with the relevance score $\geq 10$ [23]. DisGeNET is a discovery platform that contains one of the largest public collections of genes and variants related to human diseases; targets with the score $\geq 0.1$ were screened [25]. CTD is a powerful public database designed to improve people's understanding of how environmental exposure affects human health; targets with the 


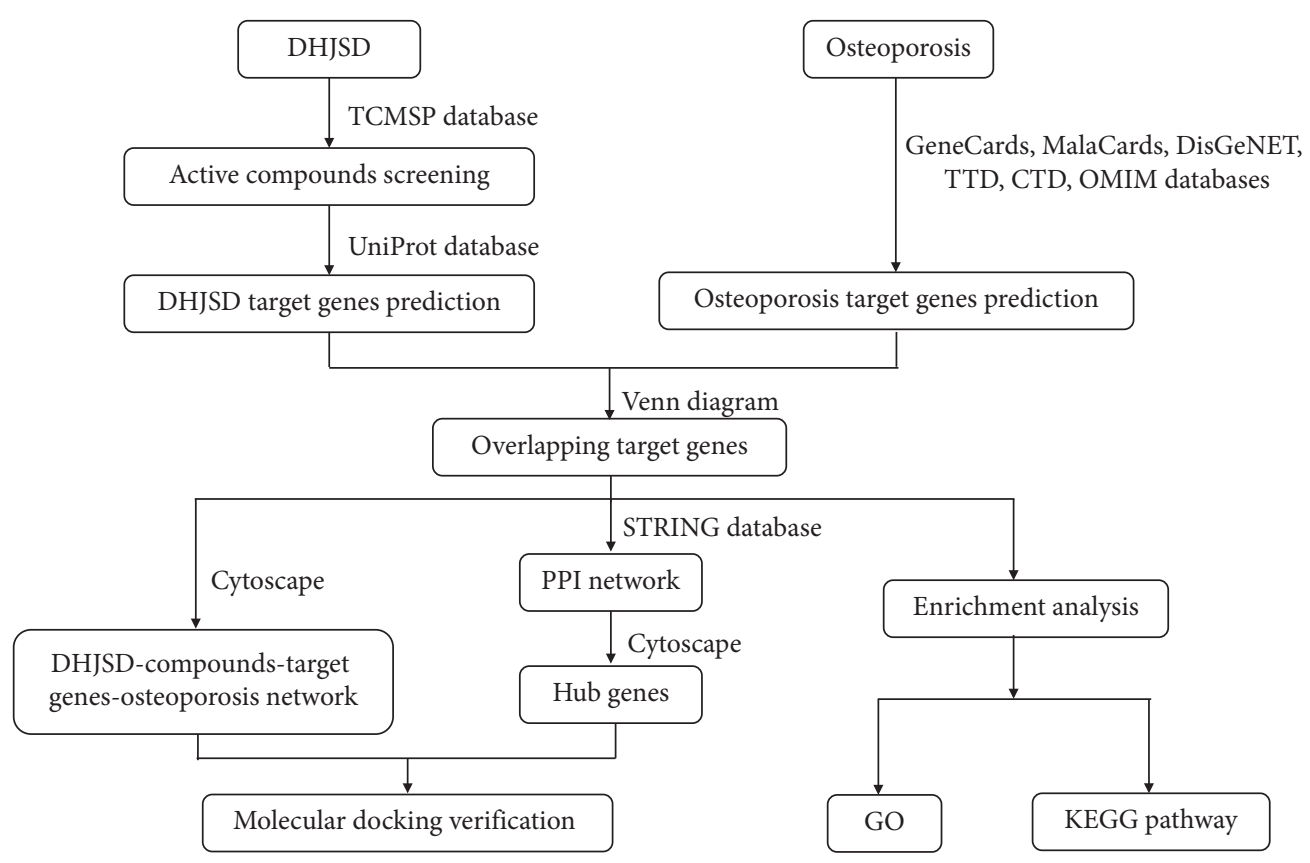

FIgURE 1: The schematic diagram of the present study to investigate potential mechanisms of DHJSD against osteoporosis.

inference score $\geq 20$ were screened [27]. Finally, the potential targets obtained from the six databases mentioned above were integrated and de-duplicated to construct a set of targets relevant to osteoporosis.

2.3. Network Construction and Analysis. DHJSD-related targets and osteoporosis-related targets were entered separately into the Venn online tool (http://www.bioinformatics. com.cn/) for common targets, which were candidate targets of DHJSD against osteoporosis. The interaction between "DHJSD-active compounds-target genes-osteoporosis" was constructed by Cytoscape software (version 3.7.2) [29].

2.4. Protein-Protein Interaction (PPI) Analysis. PPI underlies most biological processes in living cells and is essential for understanding cell physiology in normal and disease states [10]. In this study, PPI network analysis of the obtained common targets was performed using the STRING database (http://string-db.org/; version 11) with the species limited to "Homo sapiens" and a confidence score $>0.4$ [30]. The PPI networks were constructed by Cytoscape software (version 3.7.2). Additionally, the plug-in 12 CytoHubba algorithms of Cytoscape software (Degree, Maximal Clique Centrality (MCC), Density of Maximum Neighborhood Component (DMNC), Maximum Neighborhood Component (MNC), Edge Percolated Component (EPC), Closeness, Betweenness, ClusteringCoefficient, EcCentricity, Radiality, Stress, BottleNeck) were used to select the first 10 nodes for finding the hub genes [31, 32].

2.5. Gene Ontology (GO) and Kyoto Encyclopedia of Genes and Genomes (KEGG) Pathway Enrichment Analysis. We performed GO and KEGG pathway enrichment analysis using the clusterProfiler package in $R$ ( $\mathrm{R}$ 4.0.2 for Windows) to identify the biological processes and signaling pathways involved in DHJSD in treating osteoporosis [33-35]. An adjusted $P$ value of $<0.05$ was considered to identify the enriched terms.

2.6. Molecular Docking. The plug-in CytoHubba of Cytoscape software was used to screen top 10 hub genes [32]. In addition, Sankey diagram (http://sankeymatic.com/) was built with top 10 hub genes and relative active ingredients of DHJSD to find out key active ingredients. Sankey diagram discloses the relationship among herb, ingredients, and targets. Subsequently, molecular docking between top 10 hub genes and key active ingredients was carried out to predict the accuracy of the pivotal components and prediction targets using AutoDock Vina [36]. PubChem database (https://pubchem.ncbi.nlm.nih.gov/) and RCSB protein data (http://www.rcsb.org/) were selected to download the MOL2 format of ligands and PDB format of proteins [4]. Crystal of proteins were introduced to Pymol software (https://pymol.org/2/; version 2.4.1) to conduct dehydration and separation of ligands [37]. Subsequently, the crystal conducted was introduced to AutoDockTools to build a docking grid box of targets [1]. Molecular dockings were achieved via AutoDock Vina [37]. The lower Vina scores, one of the results of molecular docking, represent a more stable binding affinity of protein and ligand [38]. Eventually, the complexes of protein and compound were visualized by Pymol software.

\section{Results}

3.1. Screening of Active Compounds in DHJSD. A total of 1939 compounds in DHJSD were retrieved through the 
TCMSP database, of which 99 were from Du Huo, 46 were from Sang Ji Sheng, 27 were from Qin Jiao, 173 were from Fang Feng, 192 were from Xi Xin, 34 were from Fu Ling, 189 were from Chuan Xiong, 85 from Bai Shao, 147 from Du Zhong 190 from Ren Shen, 280 from Gan Cao, 125 from Dang Gui, 176 from Niu Xi, 76 from Shu Di Huang, and 100 from Rou Gui. According to the criteria of OB $\geq 30 \%$ and DL $\geq 0.18$, a total of 240 (after removing duplication: 209) active compounds of DHJSD were screened, of which 9 were from Du Huo, 2 were from Sang Ji Sheng, 2 were from Qin Jiao, 18 were from Fang Feng, and 8 were from Xi Xin, 15 from Fu Ling, 7 from Chuan Xiong, 13 from Bai Shao, 28 from Du Zhong, 22 from Ren Shen, 92 from Gan Cao, 2 from Dang Gui, 20 from Niu Xi, and 2 from Shu Di Huang. However, there is no compound in Rou Gui that meets the screening criteria. Table 1 shows the basic information of some active compounds in DHJSD.

3.2. Mining of the Putative Target Genes for DHJSD. By using the compound-target search function of TCMSP database, it was confirmed that 179 of the 209 active compounds in DHJSD possess target proteins. Subsequently, UniProt database was used to convert the target protein predicted by the biologically active compound of DHJSD into gene name. Finally, 267 putative target genes were identified.

3.3. Mining of the Potential Therapeutic Targets of DHJSD in Treating Osteoporosis. A total of 3131 potential therapeutic targets of osteoporosis were obtained by searching GeneCards, MalaCards, DisGeNET, TTD, CTD, and OMIM databases. The Venn diagram was drawn by using Venn online platform, which is derived from the targets regulated by the active ingredients of DHJSD and the potential targets of osteoporosis. Subsequently, a total of 205 core targets were obtained, which were the potential therapeutic targets of DHJSD in the treatment of osteoporosis, as shown in Figure 2. The "DHJSD-active compounds-target genes-osteoporosis" network of DHJSD against osteoporosis was constructed by Cytoscape software (Figure 3). In this case, the sub-network of "active compounds-target genes" includes 379 nodes and 1856 edges. We ranked the target genes according to the number of targeting nodes. Table 2 shows the basic information of some key targets of DHJSD against osteoporosis.

3.4. Construction of the PPI Network and Mining of the Hub Genes of DHJSD in Treating Osteoporosis. A total of 205 potential target genes of DHJSD in the treatment of osteoporosis were input into STRING database to obtain PPI network. The network involves 205 nodes and 4078 edges. Then, the obtained data was imported into Cytoscape 3.7.2 version for further visualization (Figure 4(a)). Subsequently, according to the 12 CytoHubba algorithms of Cytoscape software, including Degree, MCC, DMNC, MNC, EPC, Closeness, Betweenness, ClusteringCoefficient, EcCentricity, Radiality, Stress, and BottleNeck, the top 10 hub genes of DHJSD for treating osteoporosis were selected based on the above results. The top 10 genes calculated based on 12 algorithms contain a total of 41 different genes. We showed the relationship between these genes and the corresponding algorithms in the form of Chord diagrams (Figure 4(b)). Then, we sorted the genes according to the number of algorithms corresponding to them and got the top 10 hub genes, and the results were consistent with the results of the Degree algorithm (Figure 4(c)). The top 10 hub genes contained AKT1, ALB, IL6, MAPK3, VEGFA, JUN, CASP3, EGFR, MYC, and EGF. In addition, the Sankey diagram was constructed using the top 10 hub genes and the relative active compounds of DHJSD, of which MOL000098 (Quercetin) targets most hub genes (Figure 5).

3.5. GO and KEGG Pathway Enrichment Analysis. In order to elucidate the biological mechanisms of DHJSD against osteoporosis, GO and KEGG pathway enrichment analysis was performed by using clusterProfiler in R. The 205 potential targets of DHJSD for treating osteoporosis were input into the $R$, and a total of $2590 \mathrm{GO}$ terms (adjusted, $P<0.05$ ) were obtained, including 2320 biological process (BP) terms and 179 molecular function (MF) terms and 91 cellular component (CC) terms. Based on the adjusted $P$ value from small to large, the top $10 \mathrm{GO}-\mathrm{BP}$ terms were mainly enriched in cellular response to chemical stress (GO:0062197), response to metal ion (GO:0010038), response to antibiotic (GO:0046677), response to lipopolysaccharide (GO: 0032496), response to alcohol (GO:0097305), response to steroid hormone (GO:0048545), response to molecule of bacterial origin (GO:0002237), response to oxidative stress (GO:0006979), response to reactive oxygen species (GO: 0000302), and response to nutrient levels (GO:0031667). The top 10 GO-CC terms were mainly enriched in membrane raft (GO:0045121), membrane microdomain (GO:0098857), membrane region (GO:0098589), transcription regulator complex (GO:0005667), vesicle lumen (GO:0031983), RNA polymerase II transcription regulator complex (GO: 0090575), cyclin-dependent protein kinase holoenzyme complex (GO:0000307), serine/threonine protein kinase complex (GO:1902554), mitochondrial outer membrane (GO:0005741), and cytoplasmic vesicle lumen (GO: 0060205). The top 10 GO-MF terms were mainly enriched in nuclear receptor activity (GO:0004879), ligand-activated transcription factor activity (GO:0098531), steroid hormone receptor activity (GO:0003707), RNA polymerase II-specific DNA-binding transcription factor binding (GO:0061629), DNA-binding transcription factor binding (GO:0140297), "DNA-binding transcription activator activity, RNA polymerase II-specific" (GO:0001228), DNA-binding transcription activator activity (GO:0001216), phosphatase binding (GO:0019902), heme binding (GO:0020037), and NADP binding (GO:0050661). Finally, the top 10 GO enrichment terms were selected and visualized using bar diagram, as shown in Figure 6. Table 3 shows the top 10 GO enrichment items.

Additionally, a total of 170 enriched KEGG pathways (adjusted, $P<0.05$ ) were obtained, the top 10 including AGE-RAGE signaling pathway in diabetic complications 
TABLE 1: Basic information of some active compounds in DHJSD.

\begin{tabular}{|c|c|c|c|c|c|c|}
\hline Molecule ID & Molecule name & $\begin{array}{l}\text { OB } \\
(\%)\end{array}$ & $\mathrm{DL}$ & $2 \mathrm{D}$ structure & Source & $\begin{array}{l}\text { PubChem } \\
\text { CID }\end{array}$ \\
\hline MOL001924 & Paeoniflorin & 53.87 & 0.79 & & $\begin{array}{c}\text { Bai Shao (Radix Paeoniae } \\
\text { Alba) }\end{array}$ & 442534 \\
\hline MOL000492 & Cianidanol & 54.83 & 0.24 & & Bai Shao & 9064 \\
\hline MOL002135 & Myricanone & 40.60 & 0.51 & & $\begin{array}{c}\text { Chuan Xiong (Rhizoma } \\
\text { Chuanxiong) }\end{array}$ & 161748 \\
\hline MOL001494 & Ethyl linoleate & 42.00 & 0.19 & & Chuan Xiong & 5282184 \\
\hline MOL003608 & O-acetylcolumbianetin & 60.04 & 0.26 & & $\begin{array}{c}\text { Du Huo (Radix Angelicae } \\
\text { Pubescentis) }\end{array}$ & 161409 \\
\hline MOL004780 & Angelicone & 30.99 & 0.19 & & Du Huo & 616303 \\
\hline MOL002773 & Beta-carotene & 37.18 & 0.58 & & $\begin{array}{c}\text { Du Zhong (Cortex Eucommiae } \\
\text { Ulmoidis) }\end{array}$ & 5280489 \\
\hline MOL009031 & Epiquinidine & 68.22 & 0.40 & & Du Zhong & 94175 \\
\hline
\end{tabular}


TABle 1: Continued.

\begin{tabular}{|c|c|c|c|c|c|c|}
\hline Molecule ID & Molecule name & $\begin{array}{l}\text { OB } \\
(\%)\end{array}$ & $\mathrm{DL}$ & $2 \mathrm{D}$ structure & Source & $\begin{array}{c}\text { PubChem } \\
\text { CID }\end{array}$ \\
\hline MOL000296 & Hederagenin & 36.91 & 0.75 & & Fu Ling (Poria Cocos) & NA \\
\hline MOL000273 & $\begin{array}{l}\text { 16alpha- } \\
\text { Hydroxydehydrotrametenolic } \\
\text { acid }\end{array}$ & 30.93 & 0.81 & & Fu Ling & 10743008 \\
\hline MOL004328 & Naringenin & 59.29 & 0.21 & & Gan Cao (Radix Glycyrrhizae) & 439246 \\
\hline MOL000354 & Isorhamnetin & 49.60 & 0.31 & & Gan Cao & 5281654 \\
\hline MOL000392 & Formononetin & 69.67 & 0.21 & & Gan Cao & 5280378 \\
\hline MOL002714 & Baicalein & 33.52 & 0.21 & & $\begin{array}{c}\text { Niu Xi (Radix Achyranthis } \\
\text { Bidentatae) }\end{array}$ & 5281605 \\
\hline MOL000785 & Palmatine & 64.60 & 0.65 & & Niu Xi & 19009 \\
\hline MOL005344 & Ginsenoside rh2 & 36.32 & 0.56 & & Ren Shen (Panax Ginseng) & 119307 \\
\hline
\end{tabular}


Table 1: Continued.

\begin{tabular}{|c|c|c|c|c|c|c|}
\hline Molecule ID & Molecule name & $\begin{array}{l}\text { OB } \\
(\%)\end{array}$ & $\mathrm{DL}$ & $2 \mathrm{D}$ structure & Source & $\begin{array}{l}\text { PubChem } \\
\text { CID }\end{array}$ \\
\hline MOL000787 & Fumarine & 59.26 & 0.83 & & Ren Shen & 4970 \\
\hline MOL001558 & Sesamin & 56.55 & 0.83 & & Xi Xin (Herba Asari) & 72307 \\
\hline MOL002962 & 3-O-Methylviolanone & 48.23 & 0.33 & & Xi Xin & 10019512 \\
\hline MOL011753 & 5-O-Methylvisamminol & 37.99 & 0.25 & & $\begin{array}{l}\text { Fang Feng (Radix } \\
\text { Saposhnikoviae) }\end{array}$ & 441970 \\
\hline MOL000011 & Cleomiscosin A & 68.83 & 0.66 & & Fang Feng & 442510 \\
\hline MOL000449 & Stigmasterol & 43.83 & 0.76 & & $\begin{array}{c}\text { Dang Gui (Radix Angelicae } \\
\text { Sinensis), Niu Xi, Ren Shen, } \\
\text { Shu Di Huang }\end{array}$ & 5280794 \\
\hline MOL000359 & 3-Epi-beta-Sitosterol & 36.91 & 0.75 & & $\begin{array}{c}\text { Bai Shao, Chuan Xiong, Fang } \\
\text { Feng, Gan Cao, Qin Jiao, Sang } \\
\text { Ji Sheng, Shu Di Huang (Radix } \\
\text { Rehmanniae Preparata) }\end{array}$ & 12303645 \\
\hline MOL000098 & Quercetin & 46.43 & 0.28 & & $\begin{array}{l}\text { Du Zhong, Gan Cao, Sang Ji } \\
\text { Sheng (Herba Taxilli), Niu Xi }\end{array}$ & 5280343 \\
\hline MOL000358 & Beta-sitosterol & 36.91 & 0.75 & & $\begin{array}{c}\text { Bai Shao, Dang Gui, Du Huo, } \\
\text { Du Zhong, Fang Feng, Niu Xi, } \\
\text { Qin Jiao, Ren Shen }\end{array}$ & 222284 \\
\hline
\end{tabular}


TABLE 1: Continued.

\begin{tabular}{|c|c|c|c|c|c|c|}
\hline Molecule ID & Molecule name & $\begin{array}{l}\text { OB } \\
(\%)\end{array}$ & $\mathrm{DL}$ & $2 \mathrm{D}$ structure & Source & $\begin{array}{l}\text { PubChem } \\
\text { CID }\end{array}$ \\
\hline MOL000422 & Kaempferol & 41.88 & 0.24 & & $\begin{array}{c}\text { Bai Shao, Du Zhong, Gan Cao, } \\
\text { Niu Xi, Ren Shen, Xi Xin }\end{array}$ & 5280863 \\
\hline MOL000173 & Wogonin & 30.68 & 0.23 & & Fang Feng, Niu Xi & 5281703 \\
\hline MOL000211 & Mairin & 55.38 & 0.78 & & Bai Shao, Du Zhong, Gan Cao & 64971 \\
\hline MOL001942 & Isoimperatorin & 45.46 & 0.23 & & Du Huo, Fang Feng & 68081 \\
\hline MOL001941 & Ammidin & 34.55 & 0.22 & & Du Huo, Fang Feng & 10212 \\
\hline
\end{tabular}

Abbreviations: DHJSD, Duhuo Jisheng Decoction; OB, oral bioavailability; DL, drug-likeness.

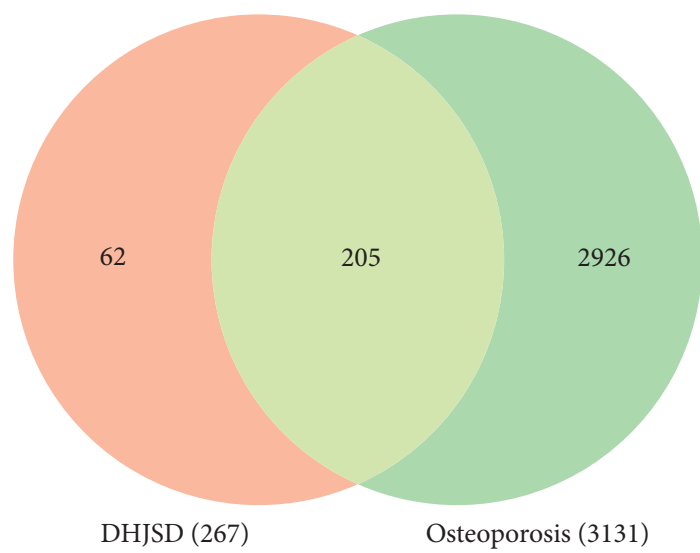

Figure 2: Venn diagram of DHJSD-related targets and osteoporosis-related targets.

(hsa04933), Kaposi sarcoma-associated herpesvirus infection (hsa05167), fluid shear stress and atherosclerosis (hsa05418), hepatitis B (hsa05161), prostate cancer (hsa05215), hepatitis C (hsa05160), pancreatic cancer (hsa05212), human cytomegalovirus infection (hsa05163), IL-17 signaling pathway (hsa04657), and TNF signaling pathway (hsa04668). The top 20 KEGG pathway enrichment terms were selected and visualized using bar diagram, as shown in Figure 7. Next, we searched the KEGG pathway database for osteoporosis, looking for potential pathways related to osteoporosis in the enriched 170 pathways. A total of 50 pathways were selected that may be associated with osteoporosis, and then we proceeded to build a network map of target genes and pathways using Cytoscape software (Figure 8). These include 13 of the pathways most strongly associated with osteoporosis, including osteoclast differentiation (hsa04380), AGE-RAGE signaling pathway in diabetic complications (hsa04933), Wnt signaling pathway (hsa04310), MAPK signaling pathway (hsa04010), apoptosis (hsa04210), chemokine signaling pathway (hsa04062), T cell receptor signaling pathway (hsa04660), B cell receptor signaling pathway (hsa04662), PI3K-Akt signaling pathway (hsa04151), JAK-STAT signaling pathway (hsa04630), 


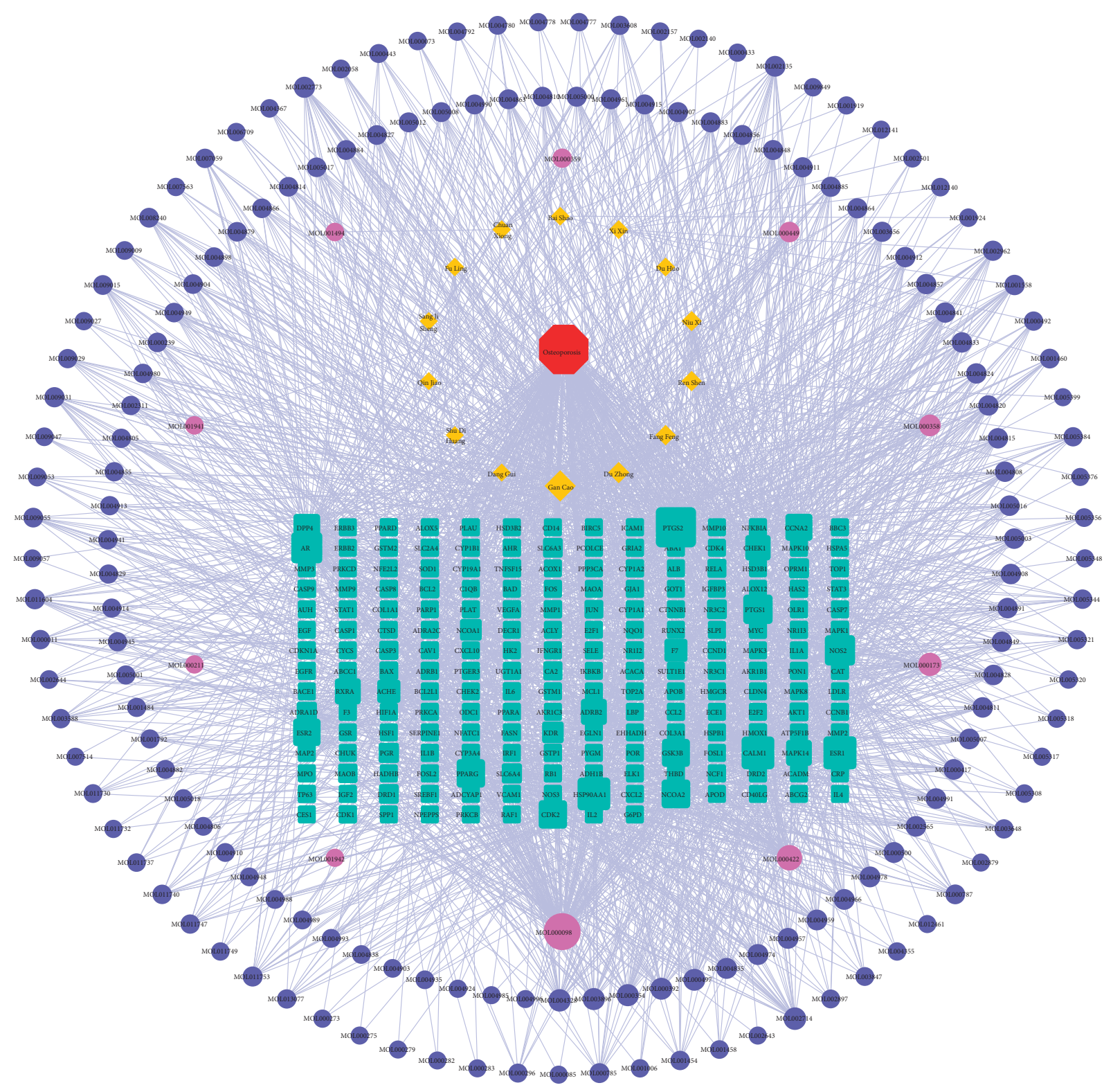

FigURE 3: "DHJSD-active compounds-target genes-osteoporosis" network. The red octagon represents the disease; the brown diamond represents the herbs contained in DHJSD; the cyan rectangle represents the potential targets; the blue ellipse belongs to the herbs contained in DHJSD; and the purple ones are common ingredients. The line between two nodes represents the interaction; the size of each node represents the number of connections.

calcium signaling pathway (hsa04020), NF-kappa B signaling pathway (hsa04064), and TNF signaling pathway (hsa04668). Table 4 shows the enriched 50 possible related pathways of osteoporosis.

3.6. Molecular Docking Results. According to the results of the Sankey diagram, we carried out molecular docking of the core target protein and active compound involved. Molecular docking between top 10 target proteins (AKT1, ALB, IL6, MAPK3, VEGFA, JUN, CASP3, EGFR, MYC, EGF) and key active compounds (Quercetin, Kaempferol, Beta-sitosterol, Wogonin, Beta-carotene, Baicalein, Naringenin, Formononetin, Paeoniflorin, Ginsenoside Rh2, and Epiquinidine) was carried out using AutoDock Vina. The docking scores of the strongest affinity of 10 core target proteins and 11 key active compounds were visualized using the heatmap, as shown in Figure 9. The binding energy between target proteins and the active compounds was approximately between -5.8 and $-10.9 \mathrm{kcal} \mathrm{mol}^{-1}$. AKT1, ALB, MAPK3, JUN, CASP3, and EGFR have stronger docking energy. The remaining target proteins also have relatively strong docking energy, which means that the compounds in DHJSD bind well to 10 core target proteins. Eventually, we chose the top 4 target protein macromolecules and small compound molecules with the best docking affinity for visualization with Pymol (Figure 10). 
TABLE 2: Basic information of some key targets of DHJSD against osteoporosis.

\begin{tabular}{|c|c|c|c|}
\hline UniProt ID & Gene symbol & Protein names & Degree \\
\hline P35354 & PTGS2 & Prostaglandin G/H synthase 2 & 144 \\
\hline $\mathrm{P} 03372$ & ESR1 & Estrogen receptor & 98 \\
\hline P0DP23 & CALM1 & Calmodulin-1 & 96 \\
\hline P07900 & HSP90AA1 & Heat shock protein HSP 90-alpha & 96 \\
\hline P10275 & AR & Androgen receptor & 85 \\
\hline P35228 & NOS2 & Nitric oxide synthase, inducible & 80 \\
\hline P23219 & PTGS1 & Prostaglandin G/H synthase 1 & 74 \\
\hline Q15596 & $\mathrm{NCOA} 2$ & Nuclear receptor coactivator 2 & 70 \\
\hline P24941 & CDK2 & Cyclin-dependent kinase 2 & 68 \\
\hline P37231 & PPARG & Peroxisome proliferator-activated receptor gamma & 68 \\
\hline P49841 & GSK3B & Glycogen synthase kinase- 3 beta & 65 \\
\hline Q92731 & ESR2 & Estrogen receptor beta & 62 \\
\hline P20248 & CCNA2 & Cyclin-A2 & 59 \\
\hline P27487 & DPP4 & Dipeptidyl peptidase 4 & 57 \\
\hline P07550 & ADRB2 & Beta-2 adrenergic receptor & 52 \\
\hline P19793 & RXRA & Retinoic acid receptor RXR-alpha & 52 \\
\hline Q16539 & MAPK14 & Mitogen-activated protein kinase 14 & 51 \\
\hline O14757 & CHEK1 & Serine/threonine-protein kinase Chk1 & 48 \\
\hline P22303 & $\mathrm{ACHE}$ & Acetylcholinesterase & 34 \\
\hline P08709 & F7 & Coagulation factor VII & 33 \\
\hline Q15788 & NCOA1 & Nuclear receptor coactivator 1 & 25 \\
\hline P35968 & KDR & Vascular endothelial growth factor receptor 2 & 24 \\
\hline P25100 & ADRA1D & Alpha-1D adrenergic receptor & 15 \\
\hline P06401 & PGR & Progesterone receptor & 15 \\
\hline P31645 & SLC6A4 & Sodium-dependent serotonin transporter & 15 \\
\hline P35372 & OPRM1 & $\mathrm{Mu}$-type opioid receptor & 14 \\
\hline P21728 & DRD1 & $\mathrm{D}(1 \mathrm{~A})$ dopamine receptor & 12 \\
\hline P08235 & NR3C2 & Mineralocorticoid receptor & 12 \\
\hline Q01959 & SLC6A3 & Sodium-dependent dopamine transporter & 12 \\
\hline P27338 & MAOB & Amine oxidase [flavin-containing] B & 10 \\
\hline Q92934 & BCL2 & Bcl2-associated agonist of cell death & 8 \\
\hline P00918 & CA2 & Carbonic anhydrase 2 & 8 \\
\hline P42574 & CASP3 & Caspase- 3 & 8 \\
\hline Q04206 & RELA & Transcription factor p65 & 7 \\
\hline P31749 & AKT1 & RAC-alpha serine/threonine-protein kinase & 6 \\
\hline Q07812 & BAX & Apoptosis regulator BAX & 6 \\
\hline P05412 & JUN & Transcription factor AP-1 & 6 \\
\hline O95150 & TNFSF15 & Tumor necrosis factor ligand superfamily member 15 & 5 \\
\hline P55211 & CASP9 & Caspase -9 & 4 \\
\hline P24385 & CCND1 & G1/S-specific cyclin-D1 & 4 \\
\hline P03956 & MMP1 & Interstitial collagenase & 4 \\
\hline P08588 & ADRB1 & Beta-1 adrenergic receptor & 3 \\
\hline P35869 & AHR & Aryl hydrocarbon receptor & 3 \\
\hline P15121 & AKR1B1 & Aldo-keto reductase family 1 member $\mathrm{B} 1$ & 3 \\
\hline Q14790 & CASP8 & Caspase- 8 & 3 \\
\hline P06493 & CDK1 & Cyclin-dependent kinase 1 & 3 \\
\hline P05177 & CYP1A2 & Cytochrome P450 1A2 & 3 \\
\hline P08684 & CYP3A4 & Cytochrome P450 3A4 & 3 \\
\hline P09211 & GSTP1 & Glutathione S-transferase P & 3 \\
\hline Q92819 & HAS2 & Hyaluronan synthase 2 & 3 \\
\hline P09601 & HMOX1 & Heme oxygenase 1 & 3 \\
\hline P05231 & IL6 & Interleukin-6 & 3 \\
\hline P28482 & MAPK1 & Mitogen-activated protein kinase 1 & 3 \\
\hline P04150 & $\mathrm{NR} 3 \mathrm{C} 1$ & Glucocorticoid receptor & 3 \\
\hline Q03181 & PPARD & Peroxisome proliferator-activated receptor delta & 3 \\
\hline $\mathrm{P} 14672$ & SLC2A4 & Solute carrier family 2 , facilitated glucose transporter member 4 & 3 \\
\hline P00441 & SOD1 & Superoxide dismutase $[\mathrm{Cu}-\mathrm{Zn}]$ & 3 \\
\hline Q9H3D4 & TP63 & Tumor protein 63 & 3 \\
\hline P15692 & VEGFA & Vascular endothelial growth factor A & 3 \\
\hline Q13085 & ACACA & Acetyl-CoA carboxylase 1 & 2 \\
\hline
\end{tabular}


TABLE 2: Continued.

\begin{tabular}{lccc}
\hline UniProt ID & Gene symbol & Protein names & Degree \\
\hline P00533 & EGFR & Epidermal growth factor receptor & 2 \\
P01106 & MYC & Myc proto-oncogene protein & 2 \\
P02768 & ALB & Albumin & 1 \\
P01133 & EGF & Pro-epidermal growth factor & 1 \\
P27361 & MAPK3 & Mitogen-activated protein kinase 3 & 1 \\
\hline
\end{tabular}
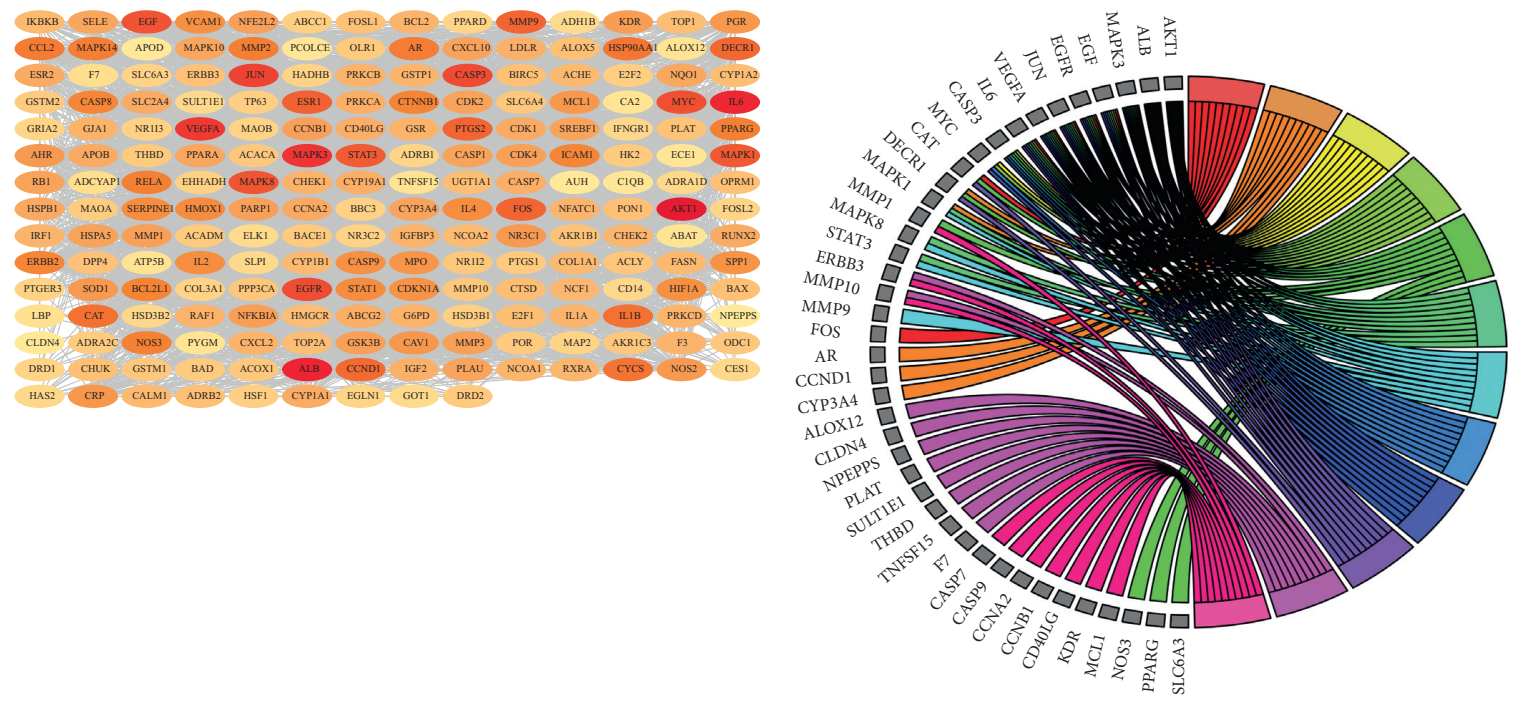

$\begin{array}{ll}\text { Algorithms } & \\ \square \text { Betweenness } & \square \text { Closeness } \\ \square \text { Eccentricity } & \square \text { MCC } \\ \square \text { Radiality } & \square \text { Clustering coefficient } \\ \square \text { Bottle neck } & \square \text { Degree } \\ \square \text { EPC } & \square \text { MNC } \\ \square \text { Stress } & \square \text { DMNC }\end{array}$

(a)

(b)

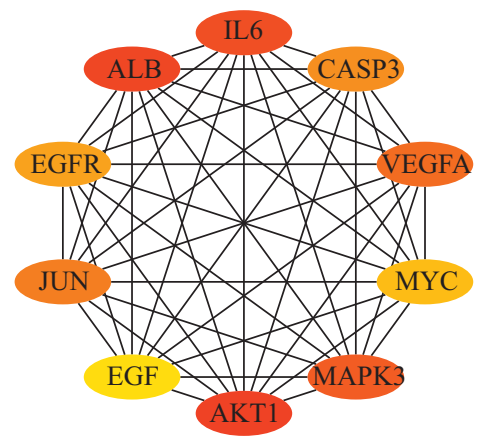

(c)

FIgURE 4: The PPI network of potential targets of DHJSD in the treatment of osteoporosis. (a) The PPI network from STRING was further analyzed using Cytoscape software (the line between two nodes indicates the interaction. The darker the color of the node, the better the relationship between them). (b) Chord diagram of the corresponding relationship between the top 10 genes and 12 CytoHubba algorithms. (c) The top 10 hub genes were identified by Degree (the darker the color of the node, the greater the degree).

\section{Discussion}

Osteoporosis is a degenerative disease, which increases with age. With the prolongation of human life and the coming of aging society, osteoporosis has become an important health problem [1]. Considering the threat of the aging of the global population and the increasing side effects of clinical drugs, finding potential drugs for the prevention and treatment of osteoporosis from natural products is a novel treatment strategy [2]. DHJSD is an extremely common TCM that has 


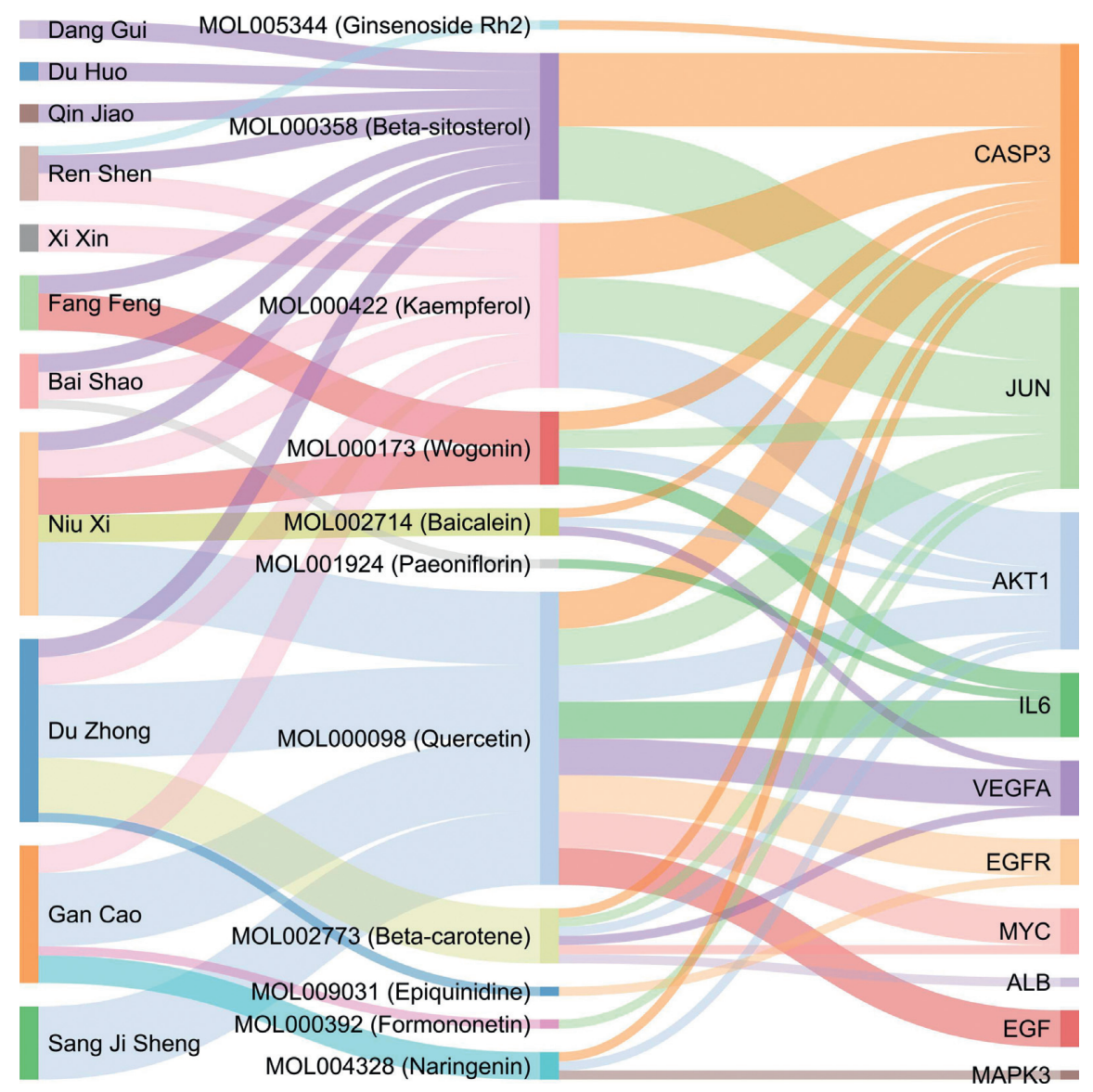

Figure 5: Sankey diagram. The colored vertical bars on the left represent the different herbs contained in DHJSD, the middle bars represent the active ingredients of the herbs, and the right bars represent the target genes. The size of the bars and the thickness of the lines represent the number of interactions.

been used in China to treat patients with osteoporosis, especially postmenopausal osteoporosis [2]. However, the mechanism of action of TCM is usually elusive due to the ingredients it contains. It is of great significance to explore the molecular mechanism of TCM by using systematic and normative bioinformatics methods to mine multiple databases to integrate and analyze the information of target proteins and compounds [4]. A systematic network pharmacological method for determining molecular biological networks has been developed, which may be used to discover new therapeutic effects of drugs derived from medicinal plants. In this study, based on systematic network pharmacology, including ADME system assessment, PPI network analysis, GO and KEGG pathway analysis, and molecular docking verification, the active ingredients and potential targets of DHJSD in the treatment of osteoporosis were evaluated.

According to the principle of ADME (setting $\mathrm{OB} \geq 30$ and $\mathrm{DL} \geq 0.18$ ) [20], we searched the TCMSP database and screened a total of 209 active compounds (after deduplication) from the 15 herbs contained in DHJSD (Rou Gui did not find a compound that meets the requirements). By using the compound-target search function of the TCMSP database, 267 target proteins were finally found from 209 active compounds (30 of which did not find the corresponding target protein). Then, we transformed the full name of the target protein into the gene ID through UniProt database. Through searching 6 disease-target databases (GeneCards, MalaCards, DisGeNET, TTD, CTD, and OMIM), a total of 3131 potential osteoporosis treatment targets were obtained. Finally, we obtained 205 potential target genes of DHJSD in the treatment of osteoporosis. The network of DHJSD against osteoporosis was built, which was involved in 394 nodes and 2265 interactions. Furthermore, PPI network has been constructed involved in 205 nodes and 4078 edges. Top 10 hub genes were revealed by weighing the CytoHubba 12 algorithms of Cytoscape software (Degree, MCC, DMNC, MNC, EPC, Closeness, Betweenness, ClusteringCoefficient, EcCentricity, Radiality, Stress, and BottleNeck), including AKT1, ALB, IL6, MAPK3, VEGFA, JUN, CASP3, EGFR, MYC, and EGF.

AKT1 is one of three closely related serine/threonine protein kinases (AKT1, AKT2, and AKT3), which regulates many physiological processes including metabolism, proliferation, cell survival, and angiogenesis [39]. A study published in 2012 showed that AKT1 may be a regulator of the differentiation and function of osteoblasts and osteoclasts [40]. IL6 is an important inflammatory factor, and its function involves a variety of inflammation-related disease states, including diabetes mellitus susceptibility and systemic juvenile rheumatoid arthritis [41]. A study published in 2018 


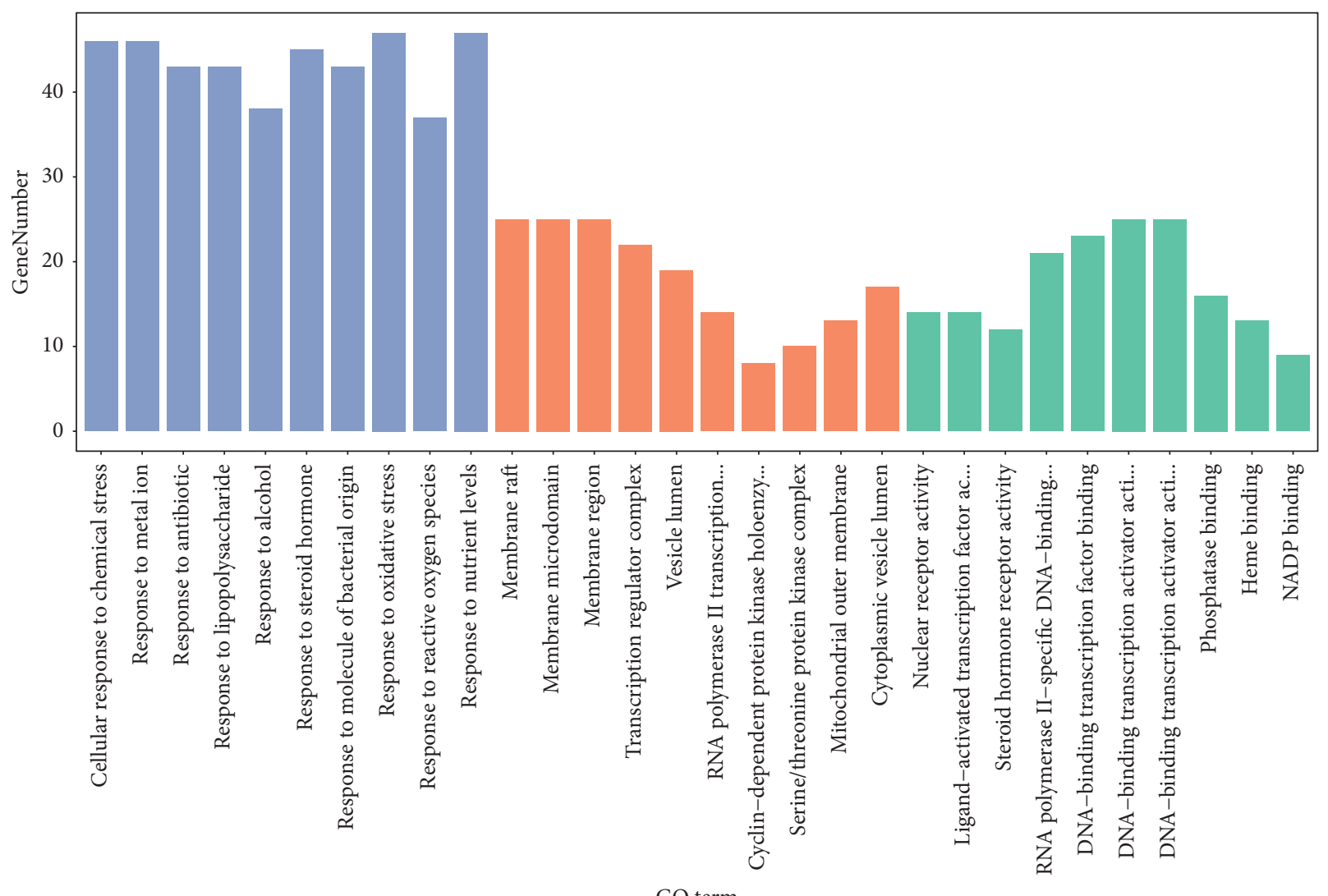

GO term

Biological process

Cellular component

Molecular function

Figure 6: Barplot of the top $10 \mathrm{GO}$ enrichment items. The GO enrichment items (BP, CC, MF) are arranged from left to right according to the adjusted $P$ value.

showed that the upregulation of IL6 expression is an important factor in promoting the osteogenic differentiation of adipose-derived stem cells [42]. ALB is the most important protein in human plasma, which maintains the body's nutrition and osmotic pressure, and acts as a carrier protein for a variety of endogenous molecules (including hormones, fatty acids, and metabolites) and exogenous drugs [43]. A retrospective study suggested that preoperative ALB levels may help predict complications after osteoporotic vertebral compression fractures [43]. MAPK3 is a member of the MAP kinase family and plays a role in the signal cascade that responds to various extracellular signals to regulate various cellular processes, such as proliferation, differentiation, and cell cycle progression [44]. Studies have shown that MAPK3 promotes the expression of RUNX2, and targeting MAPK3 can affect osteoblast differentiation [44]. VEGFA is a member of the PDGF/VEGF growth factor family, which is active in angiogenesis, angiogenesis, and endothelial cell growth, and induces endothelial cell proliferation, promotes cell migration, and inhibits cell apoptosis [45]. By inhibiting the expression of VEGFA, miR-16-5p could exert an antiosteogenesis effect [45]. The human JUN gene encodes a protein that is highly similar to the viral protein, which directly interacts with specific target DNA sequences to regulate gene expression [46]. By activating the c-Fos/c-Jun pathway, IL-7/IL-7R could promote RANKL-mediated osteoclast formation and bone resorption and induce bone loss in ovariectomized mice [46]. The protein encoded by the CASP3 gene is a cysteine-aspartic acid protease, which plays a vital role in the execution-phase of cell apoptosis [47]. By targeting CASP3 and activating the PI3K-Akt signaling pathway, the overexpression of miR-378 could attenuate the osteogenic differentiation inhibited by high glucose [47]. The protein encoded by the EGF gene is a member of the epidermal growth factor superfamily, which acts as an effective mitogenic factor and plays an important role in the growth, proliferation, and differentiation of various cell types [48]. EGFR gene encodes a protein that is a receptor for members of the epidermal growth factor family [48]. Studies have shown that inhibition of the EGFR signaling pathway inhibits the expression of the enhancer of zeste homolog 2 (Ezh2) through the ERK1/2 pathway, thereby promoting the senescence of osteoprogenitor cells [48]. MYC is a protooncogene and encodes a nuclear phosphoprotein, which plays a role in cell cycle progression, apoptosis, and cell transformation [49]. In a mouse model of osteoporosis, both 
Table 3: The top 10 Gene Ontology (GO) enrichment items.

\begin{tabular}{|c|c|c|c|c|c|}
\hline ID & Description & $P$ value & $\begin{array}{l}\text { Adjust } \\
P \text { value }\end{array}$ & $\begin{array}{c}\text { Gene } \\
\text { number }\end{array}$ & GO items \\
\hline GO:0062197 & Cellular response to chemical stress & $2.20332 E-36$ & $9.75 E-33$ & 46 & Biological process \\
\hline GO:0010038 & Response to metal ion & $1.04408 E-35$ & $2.31 E-32$ & 46 & Biological process \\
\hline GO:0046677 & Response to antibiotic & $5.44269 E-34$ & $8.03 E-31$ & 43 & Biological process \\
\hline GO:0032496 & Response to lipopolysaccharide & $8.0729 E-34$ & $8.93 E-31$ & 43 & Biological process \\
\hline GO:0097305 & Response to alcohol & $1.19658 E-33$ & $1.06 E-30$ & 38 & Biological process \\
\hline GO:0048545 & Response to steroid hormone & $2.14139 E-33$ & $1.58 E-30$ & 45 & Biological process \\
\hline GO:0002237 & Response to molecule of bacterial origin & $4.25534 E-33$ & $2.69 E-30$ & 43 & Biological process \\
\hline GO:0006979 & Response to oxidative stress & $1.67651 E-32$ & $9.27 E-30$ & 47 & Biological process \\
\hline GO:0000302 & Response to reactive oxygen species & $2.14509 E-32$ & $1.05 E-29$ & 37 & Biological process \\
\hline GO:0031667 & Response to nutrient levels & $1.65363 E-30$ & $7.32 E-28$ & 47 & Biological process \\
\hline GO:0004879 & Nuclear receptor activity & $1.2238 E-16$ & $3.33 E-14$ & 14 & $\begin{array}{l}\text { Molecular } \\
\text { function }\end{array}$ \\
\hline GO:0098531 & Ligand-activated transcription factor activity & $1.2238 E-16$ & $3.33 E-14$ & 14 & $\begin{array}{l}\text { Molecular } \\
\text { function }\end{array}$ \\
\hline GO:0003707 & Steroid hormone receptor activity & $1.51056 E-12$ & $2.74 E-10$ & 12 & $\begin{array}{l}\text { Molecular } \\
\text { function }\end{array}$ \\
\hline GO:0061629 & $\begin{array}{l}\text { RNA polymerase II-specific DNA-binding transcription } \\
\text { factor binding }\end{array}$ & $1.0407 E-11$ & $1.42 E-09$ & 21 & $\begin{array}{l}\text { Molecular } \\
\text { function }\end{array}$ \\
\hline GO:0140297 & DNA-binding transcription factor binding & $2.02886 E-11$ & $2.21 E-09$ & 23 & $\begin{array}{l}\text { Molecular } \\
\text { function }\end{array}$ \\
\hline GO:0001228 & $\begin{array}{c}\text { DNA-binding transcription activator activity, RNA } \\
\text { polymerase II-specific }\end{array}$ & $5.32777 E-11$ & $4.35 E-09$ & 25 & $\begin{array}{l}\text { Molecular } \\
\text { function }\end{array}$ \\
\hline GO:0001216 & DNA-binding transcription activator activity & $5.59254 E-11$ & $4.35 E-09$ & 25 & $\begin{array}{l}\text { Molecular } \\
\text { function }\end{array}$ \\
\hline GO:0019902 & Phosphatase binding & $4.84351 E-10$ & $3.29 E-08$ & 16 & $\begin{array}{l}\text { Molecular } \\
\text { function }\end{array}$ \\
\hline GO:0020037 & Heme binding & $5.8588 E-09$ & $3.54 E-07$ & 13 & $\begin{array}{l}\text { Molecular } \\
\text { function }\end{array}$ \\
\hline GO:0050661 & NADP binding & $7.53723 E-09$ & $4.10 E-07$ & 9 & $\begin{array}{l}\text { Molecular } \\
\text { function }\end{array}$ \\
\hline GO:0045121 & Membrane raft & $3.10297 E-15$ & $5.63 E-13$ & 25 & $\begin{array}{l}\text { Cellular } \\
\text { component }\end{array}$ \\
\hline GO:0098857 & Membrane microdomain & $3.33964 E-15$ & $5.63 E-13$ & 25 & $\begin{array}{l}\text { Cellular } \\
\text { component }\end{array}$ \\
\hline GO:0098589 & Membrane region & $7.90284 E-15$ & $8.88 E-13$ & 25 & $\begin{array}{l}\text { Cellular } \\
\text { component }\end{array}$ \\
\hline GO:0005667 & Transcription regulator complex & $4.33831 E-10$ & $3.66 E-08$ & 22 & $\begin{array}{l}\text { Cellular } \\
\text { component }\end{array}$ \\
\hline GO:0031983 & Vesicle lumen & $1.59056 E-09$ & $1.07 E-07$ & 19 & $\begin{array}{l}\text { Cellular } \\
\text { component }\end{array}$ \\
\hline GO:0090575 & RNA polymerase II transcription regulator complex & $2.17645 E-09$ & $1.22 E-07$ & 14 & $\begin{array}{l}\text { Cellular } \\
\text { component }\end{array}$ \\
\hline GO:0000307 & Cyclin-dependent protein kinase holoenzyme complex & $1.03807 E-08$ & $5.00 E-07$ & 8 & $\begin{array}{l}\text { Cellular } \\
\text { component }\end{array}$ \\
\hline GO:1902554 & Serine/threonine protein kinase complex & $2.64297 E-08$ & $1.11 E-06$ & 10 & $\begin{array}{l}\text { Cellular } \\
\text { component }\end{array}$ \\
\hline GO:0005741 & Mitochondrial outer membrane & $4.65534 E-08$ & $1.74 E-06$ & 13 & $\begin{array}{l}\text { Cellular } \\
\text { component }\end{array}$ \\
\hline GO:0060205 & Cytoplasmic vesicle lumen & $5.57446 E-08$ & $1.88 E-06$ & 17 & $\begin{array}{l}\text { Cellular } \\
\text { component }\end{array}$ \\
\hline
\end{tabular}

the loss of MYC and the pharmacological inhibitory effect of $\mathrm{ERR} \alpha$ reduced bone loss [49]. The main physiological processes regulated by proteins encoded by the top 10 target genes include inflammatory response, immune response, cell proliferation, differentiation, apoptosis, migration, cell cycle progression, endocrine metabolism, angiogenesis, growth, and nutrition. Based on the above analysis results, it is also speculated that DHJSD against osteoporosis may play a role through the above process.

The one-to-one correspondence between the top $10 \mathrm{hub}$ genes and the corresponding active compounds contained in DHJSD had been shown using the Sankey diagram. The relative active compounds include MOL000098 (Quercetin), MOL000422 (Kaempferol), MOL000358 (Beta-sitosterol), 


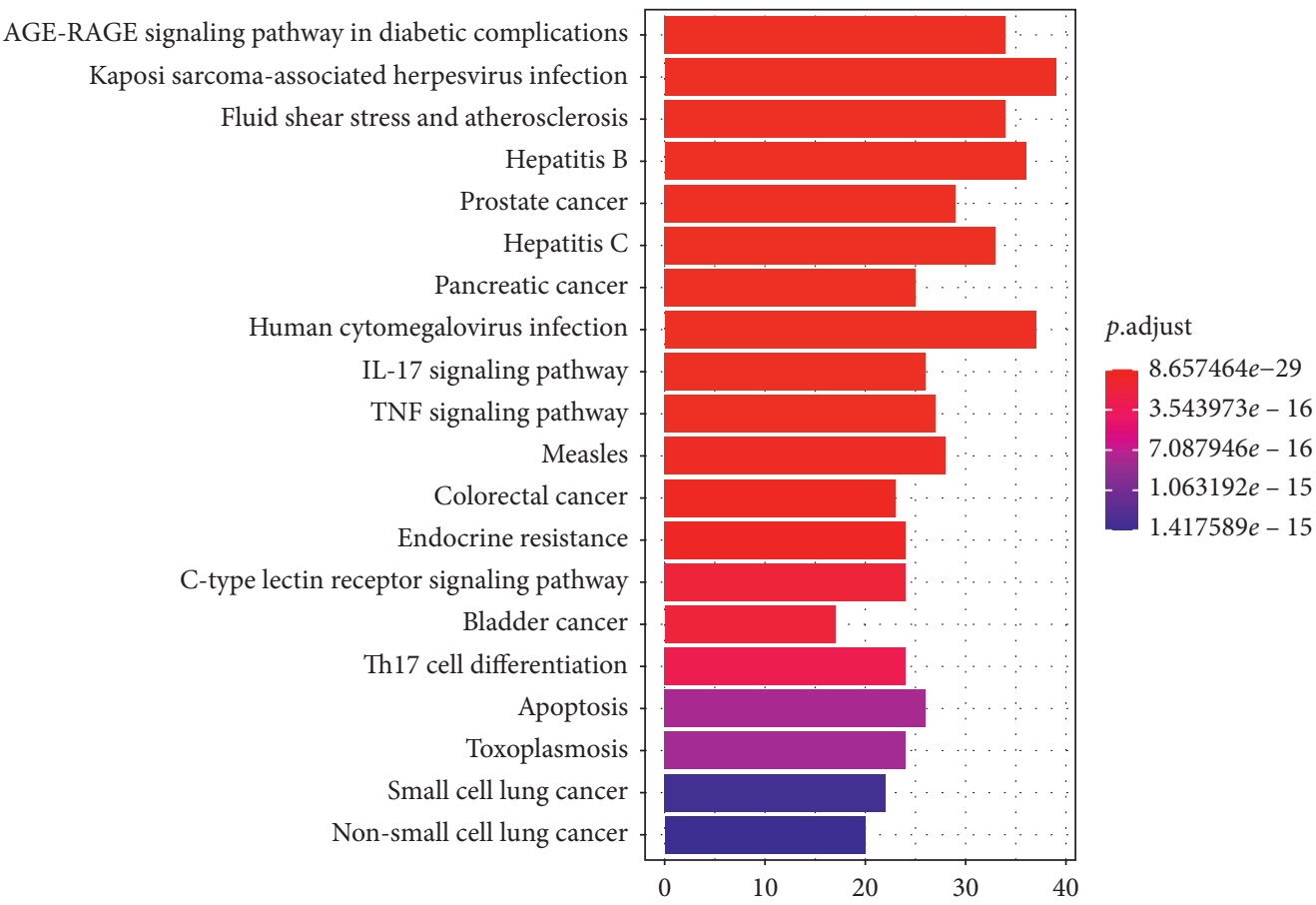

Figure 7: Barplot of the top 20 KEGG pathway enrichment items. The color of each bar represents the adjusted $P$ value, and the length represents the number of enriched genes.

MOL000173 (Wogonin), MOL002773 (Beta-carotene), MOL002714 (Baicalein), MOL004328 (Naringenin), MOL000392 (Formononetin), MOL001924 (Paeoniflorin), MOL005344 (Ginsenoside Rh2), and MOL009031 (Epiquinidine). Among them, Quercetin targets most central genes. Quercetin is a member of the flavonoid family isolated from onion, apple, grape, tea, and many kinds of Chinese herbal medicines, which seems to have obvious antiosteoporosis properties [50]. Studies have shown that Quercetin alone or in combination with alendronate could prevent glucocorticoid-induced osteoporosis through its bone formation stimulation [50]. Quercetin promotes bone marrow mesenchymal stem cells (BMSCs) proliferation and osteogenic differentiation, improves the in vitro model of osteoporosis, and provides protection against TNF- $\alpha$-induced impairment of BMSC osteogenic function [51].

Then, we performed the GO and KEGG pathway enrichment analysis of genes that DHJSD against osteoporosis. Based on the adjusted $P$ value from small to large, the top 3 GO-BP terms were mainly enriched in cellular response to chemical stress, response to metal ion, and response to antibiotic. The top $3 \mathrm{GO}-\mathrm{CC}$ terms were mainly enriched in membrane raft, membrane microdomain, and membrane region. The top 3 GO-MF terms were mainly enriched in nuclear receptor activity, ligand-activated transcription factor activity, and steroid hormone receptor activity. Based on the adjusted $P$ value from small to large, the top 10 KEGG pathways were mainly enriched in AGE-RAGE signaling pathway in diabetic complications, Kaposi sarcoma-associated herpesvirus infection, fluid shear stress, and atherosclerosis, hepatitis $\mathrm{B}$, prostate cancer, hepatitis $\mathrm{C}$, pancreatic cancer, human cytomegalovirus infection, and IL-17 signaling pathway. We searched for osteoporosis in the KEGG pathway database and found that there are ten pathways directly related to osteoporosis and related diseases, including osteoclast differentiation (hsa04380), AGE-RAGE signaling pathway in diabetic complications (hsa04933), Wnt signaling pathway (hsa04310), MAPK signaling pathway (hsa04010), apoptosis (hsa04210), chemokine signaling pathway (hsa04062), T cell receptor signaling pathway (hsa04660), B cell receptor signaling pathway (hsa04662), endocrine and other factor-regulated calcium reabsorption (hsa04961), and mineral absorption (hsa04978). The first eight pathways are in the list of KEGG pathway enrichment analysis we have done. Six of the top 10 hub genes (AKT1, MAPK3, JUN, CASP3, IL6, and VEGFA) are enriched in AGE-AGE signaling pathway in diabetic complications, three of the top 10 hub genes (AKT1, MAPK3, and JUN) are enriched in osteoclast differentiation, eight of the top 10 hub genes (AKT1, MAPK3, VEGFA, JUN, CASP3, EGFR, MYC, and EGF) are enriched in MAPK signaling pathway, two of the top 10 hub genes (JUN, MYC) are enriched in Wnt signaling pathway, four of the top 10 hub genes (AKT1, MAPK3, JUN, and CASP3) are enriched in Apoptosis, two of the top 10 hub genes (AKT1, MAPK3) are enriched in Chemokine signaling pathway, and three of the top 10 hub genes (AKT1, MAPK3, and JUN) are enriched in T cell receptor signaling pathway and $\mathrm{B}$ cell receptor signaling pathway.

In addition, the enriched pathways related to the above pathways in the KEGG database include PI3K-Akt signaling pathway (hsa04151; enriched genes: AKT1, IL6, MAPK3, VEGFA, EGFR, MYC, EGF), JAK-STAT signaling pathway (hsa04630; enriched genes: AKT1, IL6, EGFR, MYC, EGF), 


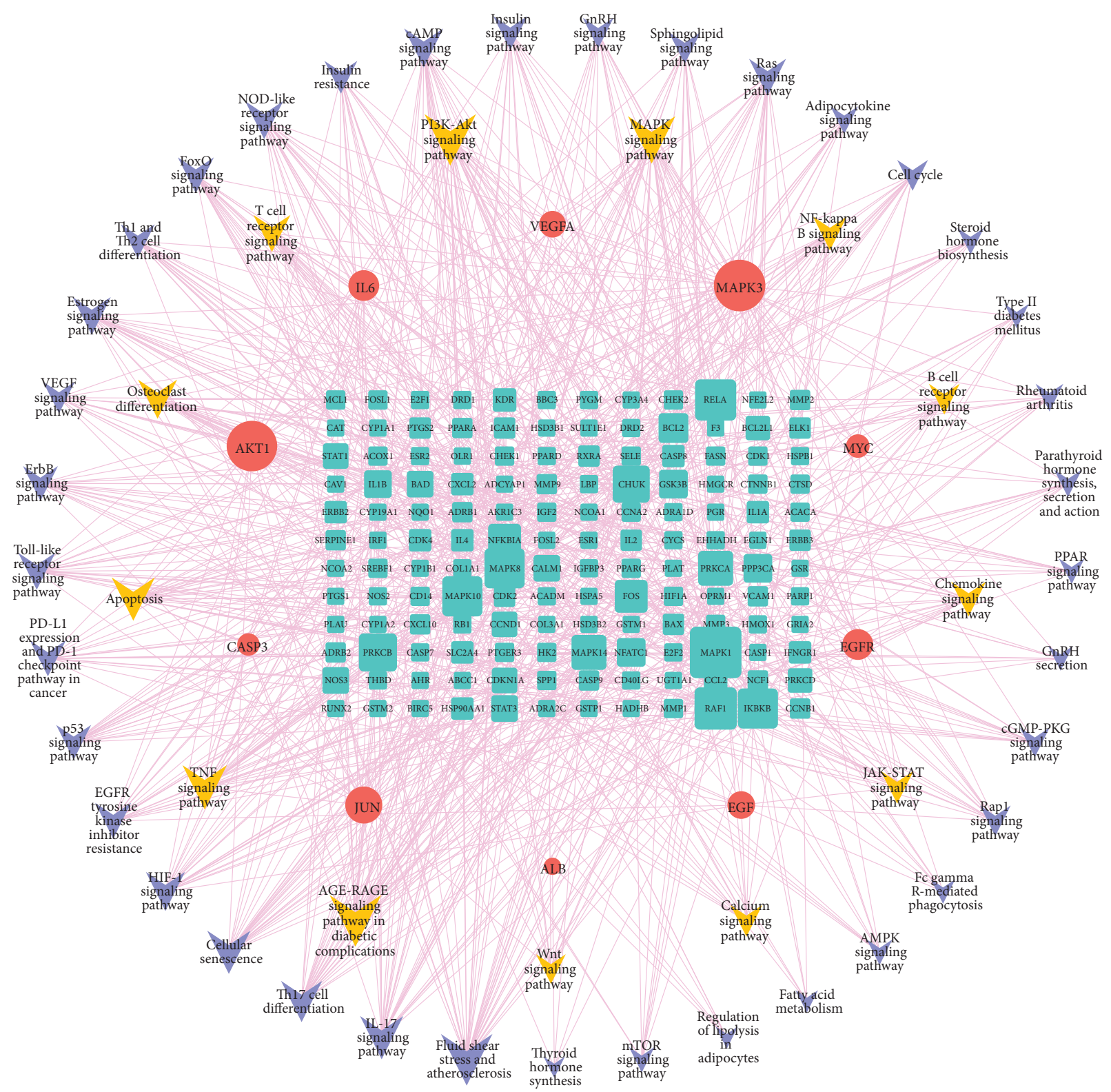

FIGURE 8: "Target genes-pathways" network. The cyan ellipse represents the potential targets of DHJSD against osteoporosis; the red ellipse represents the top 10 hub genes of DHJSD against osteoporosis; the brown arrow represents potential osteoporosis-related pathways; the light blue arrow represents osteoporosis-related pathways. The line between two nodes represents the interaction; the size of each node represents the number of connections. The lines consistent with the color of each hub gene represent the pathways enriched by that gene.

calcium signaling pathway (hsa04020; enriched genes: EGFR, EGF), TNF signaling pathway (hsa04668; enriched genes: AKT1, IL6, MAPK3, JUN, and CASP3), and NFkappa B signaling pathway (hsa04064). Li et al. [52] found that, through the PI3K-Akt signaling pathway, the knockdown of LNC_000052 could promote BMSCs osteogenesis, proliferation, and migration and inhibit cell apoptosis. Studies have shown that miR-10b could promote osteogenic differentiation and increase bone formation through the TGF- $\beta$ signaling pathway [53]. In addition, JAK-STAT signaling pathway plays a role in the differentiation of osteoblasts and osteoclasts [54]. Jin et al. [55] found that sclareol prevents bone loss caused by ovariectomy in vivo by inhibiting NF- $\kappa \mathrm{B}$ and MAPK/ERK signaling pathways and inhibits osteoclast production in vitro. Zha et al. [56] found that miR-920 targets HOXA7 through the MAPK signaling pathway to promote the osteogenic differentiation of human bone mesenchymal stem cells. It is well known that there are many pathways that have a large or small relationship with osteoporosis, and these pathways are classified mainly as bone metabolism, inflammatory response, immune response, endocrine system, and cell apoptosis [57].

By mining the database, we obtained the compounds contained in each herb in DHJSD, and then we predicted the potential targets of DHJSD by these compounds, and then we performed KEGG pathway enrichment analysis to obtain 
TABLE 4: The enriched 50 possible related pathways of osteoporosis.

\begin{tabular}{|c|c|c|c|c|}
\hline ID & Description & $P$ value & Adjust $P$ value & Gene number \\
\hline hsa04933 & AGE-RAGE signaling pathway in diabetic complications & $3.2722 E-31$ & $8.83495 E-29$ & 34 \\
\hline hsa05418 & Fluid shear stress and atherosclerosis & $8.02758 E-26$ & $6.42442 E-24$ & 34 \\
\hline hsa04657 & IL-17 signaling pathway & $2.23263 E-21$ & $6.69789 E-20$ & 26 \\
\hline hsa04668 & TNF signaling pathway & $1.93321 E-20$ & $5.21968 E-19$ & 27 \\
\hline hsa04659 & Th17 cell differentiation & $1.77974 E-17$ & $3.00331 E-16$ & 24 \\
\hline hsa04210 & Apoptosis & $5.07009 E-17$ & $8.0525 E-16$ & 26 \\
\hline hsa04218 & Cellular senescence & $1.70327 E-15$ & $1.9995 E-14$ & 26 \\
\hline hsa04066 & HIF-1 signaling pathway & $4.55414 E-15$ & $4.72929 E-14$ & 22 \\
\hline hsa01521 & EGFR tyrosine kinase inhibitor resistance & $1.19998 E-14$ & $1.11722 E-13$ & 19 \\
\hline hsa04115 & p53 signaling pathway & $3.86218 E-14$ & $3.36383 E-13$ & 18 \\
\hline hsa05235 & PD-L1 expression and PD-1 checkpoint pathway in cancer & $1.25365 E-13$ & $9.95542 E-13$ & 19 \\
\hline hsa04380 & Osteoclast differentiation & $1.52063 E-13$ & $1.14047 E-12$ & 22 \\
\hline hsa04620 & Toll-like receptor signaling pathway & $2.26415 E-13$ & $1.5283 E-12$ & 20 \\
\hline hsa04660 & $\mathrm{T}$ Cell receptor signaling pathway & $2.26415 E-13$ & $1.5283 E-12$ & 20 \\
\hline hsa04012 & ErbB signaling pathway & $6.6254 E-13$ & $4.25919 E-12$ & 18 \\
\hline hsa04151 & PI3K-akt signaling pathway & $1.24292 E-12$ & $7.80436 E-12$ & 34 \\
\hline hsa04370 & VEGF signaling pathway & $3.47037 E-12$ & $1.99361 E-11$ & 15 \\
\hline hsa04915 & Estrogen signaling pathway & $6.54943 E-12$ & $3.60403 E-11$ & 21 \\
\hline hsa04010 & MAPK signaling pathway & $6.67413 E-12$ & $3.60403 E-11$ & 30 \\
\hline hsa04658 & Th1 and Th2 cell differentiation & $3.05 E-11$ & $1.55 E-10$ & 17 \\
\hline hsa04068 & FoxO signaling pathway & $1.6511 E-10$ & $7.96064 E-10$ & 19 \\
\hline hsa04064 & NF-kappa B signaling pathway & $2.32207 E-10$ & $1.09993 E-09$ & 17 \\
\hline hsa04621 & NOD-like receptor signaling pathway & $1.2042 E-09$ & $5.16086 E-09$ & 21 \\
\hline hsa04931 & Insulin resistance & $3.58386 E-09$ & $1.423 E-08$ & 16 \\
\hline hsa04662 & B Cell receptor signaling pathway & $5.33879 E-09$ & $2.08909 E-08$ & 14 \\
\hline hsa04024 & cAMP signaling pathway & $5.66891 E-09$ & $2.1641 E-08$ & 22 \\
\hline hsa04910 & Insulin signaling pathway & $1.80579 E-08$ & $6.58868 E-08$ & 17 \\
\hline hsa04912 & GnRH signaling pathway & $2.87106 E-08$ & $1.03358 E-07$ & 14 \\
\hline hsa04071 & Sphingolipid signaling pathway & $1.04592 E-07$ & $3.62048 E-07$ & 15 \\
\hline hsa04014 & Ras signaling pathway & $4.64353 E-07$ & $1.475 E-06$ & 20 \\
\hline hsa04920 & Adipocytokine signaling pathway & $5.07934 E-07$ & $1.57635 E-06$ & 11 \\
\hline hsa04062 & Chemokine signaling pathway & $5.25301 E-07$ & $1.61172 E-06$ & 18 \\
\hline hsa04110 & Cell cycle & $1.11163 E-06$ & $3.15936 E-06$ & 14 \\
\hline hsa04630 & JAK-STAT signaling pathway & $1.15731 E-06$ & $3.25493 E-06$ & 16 \\
\hline hsa00140 & Steroid hormone biosynthesis & $1.30408 E-06$ & $3.59286 E-06$ & 10 \\
\hline hsa04930 & Type II diabetes mellitus & $9.82492 E-06$ & $2.50258 E-05$ & 8 \\
\hline hsa05323 & Rheumatoid arthritis & $1.03843 E-05$ & $2.62033 E-05$ & 11 \\
\hline hsa04928 & Parathyroid hormone synthesis, secretion and action & $3.6295 E-05$ & $8.67227 E-05$ & 11 \\
\hline hsa03320 & PPAR signaling pathway & $6.67976 E-05$ & 0.000155477 & 9 \\
\hline hsa04020 & Calcium signaling pathway & $7.36869 E-05$ & 0.000168606 & 15 \\
\hline hsa04310 & Wnt signaling pathway & $9.54755 E-05$ & 0.000213045 & 13 \\
\hline hsa04929 & GnRH secretion & 0.000116074 & 0.000254797 & 8 \\
\hline hsa04022 & cGMP-PKG signaling pathway & 0.000147423 & 0.000321001 & 13 \\
\hline hsa04015 & Rap1 signaling pathway & 0.00041579 & 0.000863564 & 14 \\
\hline hsa04666 & Fc gamma R-mediated phagocytosis & 0.00195976 & 0.003834313 & 8 \\
\hline hsa04152 & AMPK signaling pathway & 0.002019554 & 0.003922874 & 9 \\
\hline hsa01212 & Fatty acid metabolism & 0.002098286 & 0.003989699 & 6 \\
\hline hsa04923 & Regulation of lipolysis in adipocytes & 0.002098286 & 0.003989699 & 6 \\
\hline hsa04150 & mTOR signaling pathway & 0.010773083 & 0.018409699 & 9 \\
\hline hsa04918 & Thyroid hormone synthesis & 0.031361971 & 0.049810189 & 5 \\
\hline
\end{tabular}

the potential pathways of action of DHJSD based on these targets. Therefore, the above obtained targets and signaling pathways should be relevant to the functions of the herbs in DHJSD. It has been shown that the therapeutic effect of the herb $\mathrm{Du}$ Huo in osteoporotic rats is associated with the activation of $\mathrm{Wnt} / \beta$-catenin signaling pathway to promote bone formation [58]. Qin Jiao extract showed a better inhibitory effect on adjuvant arthritis rats, which may be related to the inhibition of JAK2/STAT3 signaling pathway [59]. Fu Ling significantly ameliorated renal injury in $\mathrm{db} / \mathrm{db}$ mice, and the mechanism may be related to the inhibition of p38 MAPK phosphorylation and the activation of PPAR $\gamma$ pathway [60]. These results are consistent with the functional annotations of $\mathrm{Du}$ Huo (strengthening bones and reducing back and knee pain), Qin Jiao (dispelling rheumatism), and Fu Ling (strengthening the spleen and 


\begin{tabular}{|c|c|c|c|c|c|c|c|c|c|c|}
\hline-9.80 & -8.70 & -6.70 & -9.40 & -7.00 & -8.60 & -7.90 & -8.80 & -6.20 & -6.90 & MOL000098 (Quercetin) \\
\hline-9.30 & -8.50 & -6.90 & -8.70 & -6.70 & -7.90 & -7.80 & -8.30 & -6.20 & -6.80 & MOL000422 (Kaempferol) \\
\hline-10.80 & -8.80 & -6.40 & -7.30 & -6.70 & -7.10 & -9.10 & -7.70 & -7.40 & -7.50 & MOL000358 (Beta-sitosterol) \\
\hline-9.30 & -9.00 & -6.80 & -7.10 & -6.60 & -7.50 & -7.80 & -8.00 & -6.90 & -7.00 & MOL000173 (Wogonin) \\
\hline-10.90 & -9.10 & -7.80 & -8.80 & -7.10 & -7.20 & -7.90 & -8.20 & -6.90 & -6.60 & MOL002773 (Beta-carotene) \\
\hline-9.80 & -9.10 & -7.00 & -8.50 & -6.90 & -8.20 & -8.20 & -8.00 & -6.70 & -7.20 & MOL002714 (Baicalein) \\
\hline-9.60 & -9.00 & -6.60 & -8.70 & -6.60 & -8.40 & -7.90 & -8.50 & -6.40 & -6.90 & MOL004328 (Naringenin) \\
\hline-10.20 & -8.50 & -6.80 & -8.70 & -7.20 & -7.90 & -7.90 & -8.00 & -6.80 & -7.40 & MOL000392 (Formononetin) \\
\hline-9.70 & -9.20 & -6.80 & -8.90 & -7.10 & -8.10 & -8.20 & -8.30 & -7.90 & -6.70 & MOL001924 (Paeoniflorin) \\
\hline-8.90 & -9.60 & -7.00 & -7.90 & -7.00 & -8.70 & -8.60 & -7.30 & -7.30 & -6.80 & MOL005344 (Ginsenoside Rh2) \\
\hline-9.10 & -8.60 & -6.50 & -8.50 & -5.80 & -6.70 & -7.20 & -7.30 & -6.30 & -6.80 & MOL009031 (Epiquinidine) \\
\hline 点 & 是 & $\stackrel{\circ}{\boxminus}$ & $\frac{\theta}{2}$ & 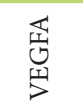 & Z & $\stackrel{n}{\stackrel{\infty}{\sharp}}$ & $\underset{\circlearrowleft}{\stackrel{\alpha}{I}}$ & $\underset{\Sigma}{\circlearrowright}$ & 岕 & \\
\hline
\end{tabular}

FIGURE 9: Heatmap of the docking scores of the active compounds of DHJSD and the target proteins.

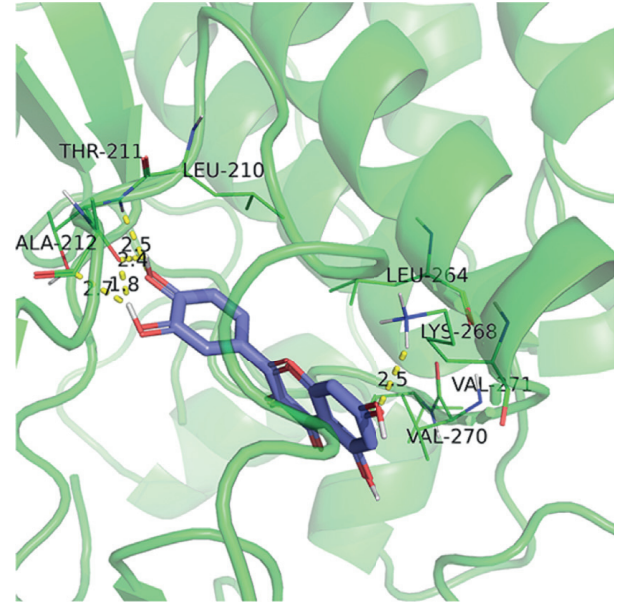

(a)

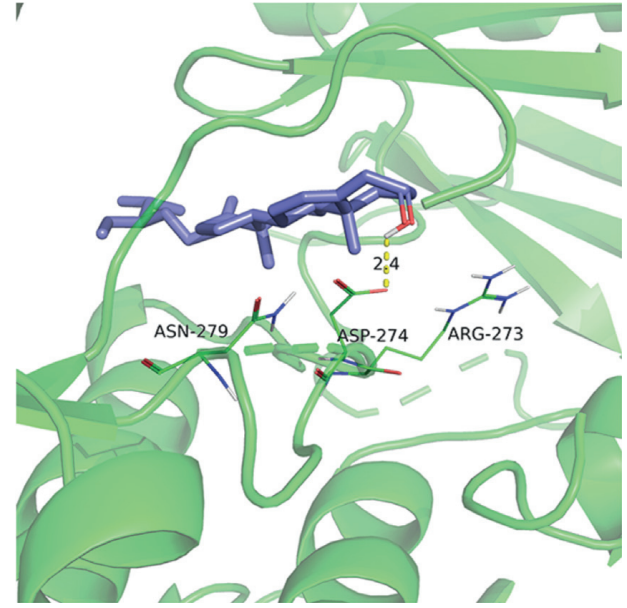

(b)

FIgURe 10: Continued. 


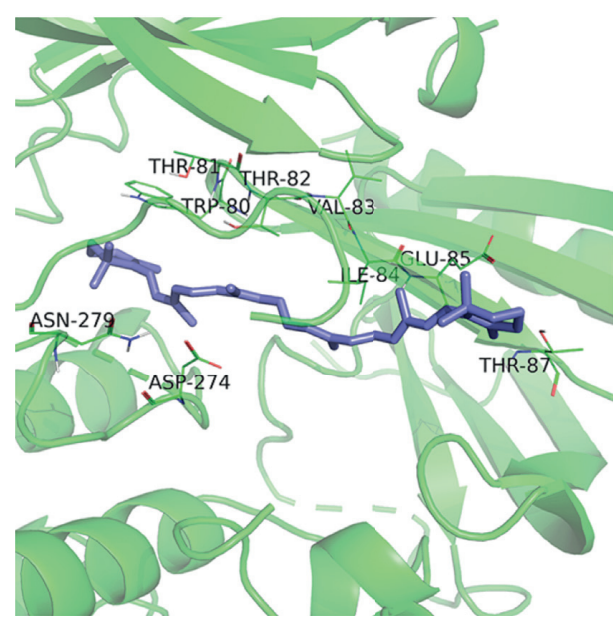

(c)

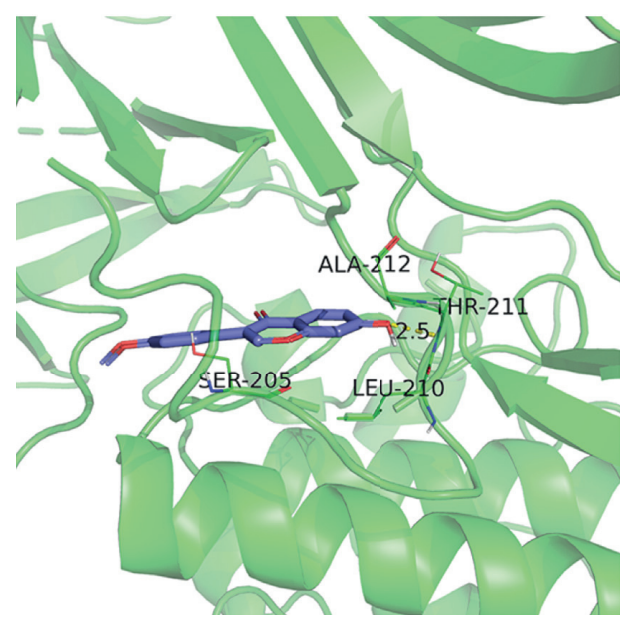

(d)

FIgUre 10: Molecular docking results of "bioactive compound-hub gene." (a) Quercetin to AKT1; (b) Beta-sitosterol to AKT1; (c) Betacarotene to AKT1; (d) Formononetin to AKT1.

benefiting the kidney) in TCM theory. The number of studies on the targets and pathways of other herbs for the treatment of osteoporosis is currently very limited, and this becomes our next research direction. By using modern pharmacological methods to explore the specific mechanisms by which DHJSD exerts its therapeutic effects, it is more beneficial to understand and complement TCM theories, and thus to uncover the essence of TCM and find alternative therapies for diseases.

\section{Conclusion}

By using the network pharmacology approach, we have studied the potential targets of DHJSD and the underlying mechanism of its anti-osteoporosis effect, which has the characteristics of multi-component, multi-target, and multi-pathway. AKT1, ALB, IL6, MAPK3, VEGFA, JUN, CASP3, EGFR, MYC, and EGF may be potential targets of DHJSD in treating osteoporosis. According to the results of enrichment analysis of KEGG pathway, we found pathways closely related to the pathological process of osteoporosis, mainly including AGE-RAGE signaling pathway in diabetic complications, osteoclast differentiation, MAPK signaling pathway, Wnt signaling pathway, PI3K-Akt signaling pathway, JAK-STAT signaling pathway, and TNF signaling pathway. Therefore, this study reveals that the anti-osteoporosis effect of DHJSD may be based on its direct or indirect regulation of the abovementioned potential targets and pathways. DHJSD may provide a promising direction for future research, so enough relevant experimental research verification is needed, which is very important for revealing its exact regulatory mechanism.

\section{Data Availability}

The data used to support the findings of this study are included within the article.

\section{Conflicts of Interest}

The authors declare that there are no conflicts of interest regarding the publication of this article.

\section{Acknowledgments}

This study was supported by the Beijing Municipal Science \& Technology Commission (grant no. Z181100001818006).

\section{References}

[1] H. Zheng, H. Feng, W. Zhang, Y. Han, and W. Zhao, "Targeting autophagy by natural product Ursolic acid for prevention and treatment of osteoporosis," Toxicology and Applied Pharmacology, vol. 409, 2020.

[2] J. Li, W. Wang, G Feng et al., "Efficacy and safety of Duhuo Jisheng decoction for postmenopausal osteoporosis: a systematic review and meta-analysis," Evidence Based Complementary and Alternative Medicine, vol. 2020, Article ID 6957825, 10 pages, 2020.

[3] J. A. Kanis, "Diagnosis of osteoporosis and assessment of fracture risk," The Lancet, vol. 359, no. 9321, p. 1929, 2002.

[4] N. Zhu and J. Hou, "Exploring the mechanism of action Xianlingubao Prescription in the treatment of osteoporosis by network pharmacology," Computational Biology and Chemistry, vol. 85, Article ID 107240, 2020.

[5] G. Li, Q. Xu, K. Han, W. Yan, and C. Huang, "Experimental evidence and network pharmacology-based analysis reveal the molecular mechanism of Tongxinluo capsule administered in coronary heart diseases," Bioscience Reports, vol. 40, no. 10, 2020.

[6] Y. Zhang, J. Jiang, H. Shen, Y. Chai, X. Wei, and Y. Xie, "Total flavonoids from Rhizoma Drynariae (Gusuibu) for treating osteoporotic fractures: implication in clinical practice," Drug Design, Development and Therapy, vol. 11, p. 1881, 2017.

[7] Z. Wu, X. Zhu, C. Xu, Y. Chen, L. Zhang, and C. Zhang, "Effect of Xianling Gubao capsules on bone mineral density in osteoporosis patients," Journal of Biological Regulators and Homeostatic Agents, vol. 31, no. 2, p. 359, 2017. 
[8] B. Xia, B. Xu, Y. Sun et al., "The effects of Liuwei Dihuang on canonical $\mathrm{Wnt} / \beta$-catenin signaling pathway in osteoporosis," Journal of Ethnopharmacology, vol. 153, no. 1, p. 133, 2014.

[9] N. Wang, H. Xin, P. Xu, Z. Yu, and D. Shou, "Erxian decoction attenuates TNF- $\alpha$ induced osteoblast apoptosis by modulating the akt/nrf2/HO-1 signaling pathway," Frontiers in Pharmacology, vol. 10, p. 988, 2019.

[10] W. Xiao, W. Sun, H. Lian, and J. Shen, "Integrated network and experimental pharmacology for deciphering the medicinal substances and multiple mechanisms of Duhuo Jisheng decoction in osteoarthritis therapy," Evidence Based Complementary and Alternative Medicine, vol. 2020, Article ID 7275057, 13 pages, 2020.

[11] Z. Xiong, P. Yi, L. Zhang, H. Ma, W. Li, and M. Tan, "Efficacy and safety of modified Duhuo Jisheng decoction in the treatment of lumbar disc herniation: a systematic review and meta-analysis," Evidence Based Complementary and Alternative Medicine, vol. 2020, Article ID 2381462, 11 pages, 2020.

[12] Y. Zhang and X. Guan, "Research situation in treatment of orthopedics diseases using Duhuo Jisheng decoction," Journal of Practical Traditional Chinese Internal Medicine, vol. 29, no. 4, p. 179, 2015.

[13] W. Liang, X. Li, and C. Li, "The core pathogenesis of postmenopausal osteoporosis-Gu Lou," Chinese Journal of Gerontology, vol. 35, no. 18, p. 5333, 2015.

[14] W. Zhang, S. Wang, R Zhang et al., "Evidence of Chinese herbal medicine Duhuo Jisheng decoction for knee osteoarthritis: a systematic review of randomised clinical trials," $B M J$ Open, vol. 6, no. 1, Article ID e008973, 2016.

[15] C.-S. Zheng, X.-J. Xu, H.-Z. Ye et al., "Computational approaches for exploring the potential synergy and polypharmacology of Duhuo Jisheng Decoction in the therapy of osteoarthritis," Molecular Medicine Reports, vol. 7, no. 6, p. 1812, 2013.

[16] Z.-C. Liu, Z.-L. Wang, C.-Y. Huang et al., "Duhuo Jisheng Decoction inhibits SDF-1-induced inflammation and matrix degradation in human degenerative nucleus pulposus cells in vitro through the CXCR4/NF- $\kappa \mathrm{B}$ pathway," Acta Pharmacologica Sinica, vol. 39, no. 6, p. 912, 2018.

[17] J. Zhao, Q. Zha, M. Jiang, H. Cao, and A. Lu, "Expert consensus on the treatment of rheumatoid arthritis with Chinese patent medicines," The Journal of Alternative and Complementary Medicine, vol. 19, no. 2, p. 111, 2013.

[18] Y. Shuai, Z. Jiang, Q. Yuan, S. Tu, and F. Zeng, "Deciphering the underlying mechanism of Eucommiae cortex against osteoporotic fracture by network pharmacology," Evidence Based Complementary and Alternative Medicine, vol. 2020, Article ID 7049812, 12 pages, 2020.

[19] J. Ru, P. Li, J Wang et al., "TCMSP: a database of systems pharmacology for drug discovery from herbal medicines," Journal of Cheminformatics, vol. 6, no. 13, 2014.

[20] K. Tsaioun, B. Blaauboer, and T. Hartung, "Evidence-based absorption, distribution, metabolism, excretion (ADME) and its interplay with alternative toxicity methods," ALTEX, vol. 33, no. 4, p. 343, 2016.

[21] S. Sheng, Z. Yang, F. Xu, and Y. Huang, "Network pharmacology-based exploration of synergistic mechanism of guanxin II formula (II) for coronary heart disease," Chinese Journal of Integrative Medicine, vol. 27, no. 2, pp. 106-114, 2020.

[22] T. UniProt Consortium, "UniProt: the universal protein knowledge base," Nucleic Acids Research, vol. 46, no. 5, p. $2699,2018$.
[23] G. Stelzer, N. Rosen, I Plaschkes et al., "The GeneCards suite: from gene data mining to disease genome sequence analyses," Current Protocols in Bioinformatics, vol. 54, pp. 1-30, 2016.

[24] N. Rappaport, M. Twik, I. Plaschkes et al., "MalaCards: an amalgamated human disease compendium with diverse clinical and genetic annotation and structured search," Nucleic Acids Research, vol. 45, no. D1, p. D877, 2017.

[25] J. Piñero, J. M. Ramírez-Anguita, J Saüch-Pitarch et al., "The DisGeNET knowledge platform for disease genomics: 2019 update," Nucleic Acids Research, vol. 48, no. D1, p. D845, 2020.

[26] Y. Wang, S. Zhang, F Li et al., "Therapeutic target database 2020: enriched resource for facilitating research and early development of targeted therapeutics," Nucleic Acids Research, vol. 48, no. D1, p. D1031, 2020.

[27] A. Davis, C. Grondin, R Johnson et al., "Comparative Toxicogenomics database (CTD): update 2021," Nucleic Acids Research, vol. 49, no. D1, pp. D1138-D1143, 2020.

[28] J. Amberger and A. Hamosh, "Searching online mendelian inheritance in man (OMIM): a knowledgebase of human genes and genetic phenotypes," Current Protocols in Bioinformatics, vol. 58, 2017.

[29] P. Shannon, A. Markiel, O Ozier et al., "Cytoscape: a software environment for integrated models of biomolecular interaction networks," Genome Research, vol. 13, no. 11, p. 2498, 2003.

[30] D. Szklarczyk, J. H. Morris, H. Cook et al., “The STRING database in 2017: quality-controlled protein-protein association networks, made broadly accessible," Nucleic Acids Research, vol. 45, no. D1, p. D362, 2017.

[31] C. Xu, R. Li, and J. Wu, "Effects of Yuanhu-Zhitong tablets on alcohol-induced conditioned place preference in mice," Biomedicine \& Pharmacotherapy, vol. 133, Article ID 110962, 2020.

[32] C. Chin, S. Chen, H. Wu, C. Ho, M. Ko, and C. Lin, "cytoHubba: identifying hub objects and sub-networks from complex interactome," BMC Systems Biology, vol. S11, 2014.

[33] Gene Ontology Consortium, "The Gene Ontology Consortium: Going forward," Nucleic Acids Research, vol. 43, Article ID D1049, 2015.

[34] M. Kanehisa, M. Araki, S Goto et al., "KEGG for linking genomes to life and the environment," Nucleic Acids Research, vol. 36, no. Database issue, p. D480, 2008.

[35] G. Yu, L.-G. Wang, Y. Han, and Q.-Y. He, "clusterProfiler: an $\mathrm{R}$ package for comparing biological themes among gene clusters," OMICS: A Journal of Integrative Biology, vol. 16, no. 5 , p. 284, 2012.

[36] O. Trott and A. J. Olson, "AutoDock Vina: improving the speed and accuracy of docking with a new scoring function, efficient optimization, and multithreading," Journal of Computational Chemistry, vol. 31, no. 2, p. 455, 2010.

[37] S. Gao, J. Sun, X. Wang, Y. Hu, Q. Feng, and X Gou, "Research on the mechanism of qushi huayu decoction in the intervention of nonalcoholic fatty liver disease based on network pharmacology and molecular docking Technology," BioMed Research International, vol. 2020, Article ID 1704960, 12 pages, 2020.

[38] J. Chang, L. Liu, Y. Wang, Y. Sui, H. Li, and L. Feng, "Investigating the multitarget mechanism of traditional Chinese medicine prescription for cancer-related pain by using network pharmacology and molecular docking approach," Evidence Based Complementary and Alternative Medicine, vol. 2020, Article ID 7617261, 11 pages, 2020. 
[39] I. Hers, E. E. Vincent, and J. M. Tavaré, "Akt signalling in health and disease," Cellular Signalling, vol. 23, no. 10, p. 1515, 2011.

[40] A. Mukherjee and P. Rotwein, "Selective signaling by Akt1 controls osteoblast differentiation and osteoblast-mediated osteoclast development," Molecular and Cellular Biology, vol. 32, no. 2, p. 490, 2012.

[41] P. F. Giampietro, C. McCarty, B. Mukesh et al., "The role of cigarette smoking and statins in the development of postmenopausal osteoporosis: a pilot study utilizing the Marshfield Clinic Personalized Medicine Cohort," Osteoporosis International, vol. 21, no. 3, p. 467, 2010.

[42] R. Wu, J. Ruan, Y Sun et al., "Long non-coding RNA HIF1AAS2 facilitates adipose derived stem cells (ASCs) osteogenic differentiation through miR-665/IL6 axis via PI3K/Akt signaling pathway," Stem Cell Research \& Therapy, vol. 9, no. 1, p. 348, 2018.

[43] A. Gupta, S. Upadhyaya, T. Cha, J. Schwab, C. Bono, and S. Hershman, "Serum albumin levels predict which patients are at increased risk for complications following surgical management of acute osteoporotic vertebral compression fractures," The Spine Journal, vol. 19, no. 11, p. 1796, 2019.

[44] Y. Yang, X. Zhou, Y Li et al., "CXCL2 attenuates osteoblast differentiation by inhibiting the ERK1/2 signaling pathway," Journal of Cell Science, vol. 132, no. 16, 2019.

[45] T. Yu, X. You, H. Zhou et al., "MiR-16-5p regulates postmenopausal osteoporosis by directly targeting VEGFA," Aging, vol. 12, no. 10, p. 9500, 2020.

[46] J.-J. Zhao, Z.-F. Wu, Y.-H. Yu, L. Wang, and L. Cheng, "Effects of interleukin-7/interleukin-7 receptor on RANKLmediated osteoclast differentiation and ovariectomy-induced bone loss by regulating c-Fos/c-Jun pathway," Journal of Cellular Physiology, vol. 233, no. 9, p. 7182, 2018.

[47] L. You, W. Gu, L. Chen, L. Pan, J. Chen, and Y. Peng, "MiR378 overexpression attenuates high glucose suppressed osteogenic differentiation through targeting CASP3 and activating PI3K/Akt signaling pathway," International Journal of Clinical and Experimental Pathology, vol. 7, no. 10, p. 7249, 2014.

[48] G. Liu, Y. Xie, J. Su et al., "The role of EGFR signaling in agerelated osteoporosis in mouse cortical bone," The FASEB Journal, vol. 33, no. 10, p. 11137, 2019.

[49] J. Lorenzo, "The many ways of osteoclast activation," Journal of Clinical Investigation, vol. 127, no. 7, p. 2530, 2017.

[50] H. Derakhshanian, M. Djalali, A. Djazayery et al., "Quercetin prevents experimental glucocorticoid-induced osteoporosis: a comparative study with alendronate," Canadian Journal of Physiology and Pharmacology, vol. 91, no. 5, p. 380, 2013.

[51] Z. Yuan, J. Min, Y Zhao et al., "Quercetin rescued TNFalpha-induced impairments in bone marrow-derived mesenchymal stem cell osteogenesis and improved osteoporosis in rats," American Journal of Translational Research, vol. 10, no. 12, p. 4313, 2018.

[52] M. Li, R. Cong, L. Yang, L. Yang, Y. Zhang, and Q. Fu, “A novel lncRNA LNC_000052 leads to the dysfunction of osteoporotic BMSCs via the miR-96-5p-PIK3R1 axis," Cell Death \& Disease, vol. 11, no. 9, p. 795, 2020.

[53] H. Li, J. Fan, L. Fan et al., "MiRNA-10b reciprocally stimulates osteogenesis and inhibits adipogenesis partly through the TGF- $\beta /$ SMAD2 signaling pathway," Aging and Disease, vol. 9, no. 6, p. 1058, 2018.

[54] A. Damerau, T. Gaber, S. Ohrndorf, and P. Hoff, "JAK/STAT activation: a general mechanism for bone development, homeostasis, and regeneration," International Journal of Molecular Sciences, vol. 21, no. 23, 2020.

[55] H. Jin, Z. Shao, Q. Wang et al., "Sclareol prevents ovariectomy-induced bone loss in vivo and inhibits osteoclastogenesis in vitro via suppressing NF- $\kappa \mathrm{B}$ and MAPK/ERK signaling pathways," Food \& Function, vol. 10, no. 10, p. 6556, 2019.

[56] J. Zha, X. Wang, and J. Di, "MiR-920 promotes osteogenic differentiation of human bone mesenchymal stem cells by targeting HOXA7," Journal of Orthopaedic Surgery and Research, vol. 15, no. 1, p. 254, 2020.

[57] W.-J. Liu, Z.-M. Jiang, Y. Chen et al., "Network pharmacology approach to elucidate possible action mechanisms of Sinomenii Caulis for treating osteoporosis," Journal of Ethnopharmacology, vol. 257, Article ID 112871, 2020.

[58] Q. Yao, N. Gao, Y. Ci, and Z. Li, "The therapeutic effect of Angelicae Pubescentis Radix on osteoporosis related to upregulating Wnt/ $\beta$-catenin signaling pathway in rats," Progress of Anatomical Sciences, vol. 25, no. 5, p. 532, 2019.

[59] L. Yang, W. Lin, and L. Deng, "Function of Gentiana macrophylla Pall on the Regulating JAK2/STAT3 signal pathway in adjuvant arthritis rats," Clinical Journal of Traditional Chinese Medicine, vol. 28, no. 6, p. 848, 2016.

[60] J. Li and D. Zhou, "Renal protection action of pachymaran and effects on p38 MAPK/PPAR- $\gamma$ signaling pathway in $\mathrm{db} /$ $\mathrm{db}$ mice," Chinese Journal of Traditional Medical Science and Technology, vol. 26, no. 3, p. 346, 2019. 